Maladies chroniques

et blessures au Canada

une publication de l'Agence

de la santé publique du Canada
Comité de rédaction de $\mathrm{MCBC}$
Howard Morrison, Ph. D.

Rédacteur scientifque en chef

Anne-Marie Ugnat, Ph. D. Rédactrice scientifique en chef déléguée

Claire Infante-Rivard, M.D., Ph. D., FRCPC Rédactrice scientifique adjointe

Barry Pless, C.M., M.D., FRCPC Rédacteur scientifique adjoint

Elizabeth Kristjansson, Ph. D. Rédactrice scientifique adjointe

Gavin McCormack, Ph. D. Rédacteur scientifique adjoint

Mylène Poulin, B. Sc., B.A. Gestionnaire de la rédaction 613-946-6963

Sylvain Desmarais, B.A., B. Ed. Gestionnaire-adjoint de la rédaction

Joanna Odrowaz-Pieniazek, B. Sc. Réviseure et correctrice d'épreuves

Anna Olivier, Ph. D. Réviseure et correctrice d'épreuves
Robert Geneau, Ph. D.

Centre de recherches pour le développement international

Brent Hagel, Ph. D.

University of Calgary

Valerie Leinan

Agence de la santé publique du Canada

Isra Levy, M.B., FRCPC, FACPM

Santé publique Ottawa

Lesli Mitchell, M.A.

Centers for Disease Control and Prevention

Scott Patten, M.D., Ph. D., FRCPC University of Calgary

Kerry Robinson, Ph. D.

Agence de la santé publique du Canada

Robert A. Spasoff, M.D. Université d'Ottawa

Richard Stanwick, M.D., FRCPC, FAAP Island Health

Ania Syrowatka, M. Sc. Université McGill

Andreas T. Wielgosz, M.D., Ph. D., FRCPC Agence de la santé publique du Canada

Russell Wilkins, M. Urb. Université d'Ottawa
Maladies chroniques et blessures au Canada (MCBC) est une revue scientifique trimestrielle présentant des données probantes récentes sur la prévention et la lutte contre les maladies chroniques (c.-à-d. non transmissibles) et les traumatismes au Canada. Selon une formule unique et depuis 1980 , la revue publie des articles soumis à une évaluation provenant des secteurs public et privé et rend compte de recherches effectuées dans des domaines tels que l'épidémiologie, la santé publique ou communautaire, la biostatistique, les sciences du comportement, et l'économie ou les services de la santé. Tous les articles de fond sont soumis à une évaluation par les pairs; les autres types d'articles ne le sont pas. Les auteurs demeurent responsables du contenu de leurs articles, et les opinions exprimées ne sont pas forcément celles du Comité de rédaction de MCBC, ni celles de l'Agence de la santé publique du Canada.

Maladies chroniques et blessures au Canada Agence de la santé publique du Canada 785, avenue Carling, Indice de l'adresse : 6806B Ottawa (Ontario) K1A 0K9

Télécopieur : 613-941-2057 Courriel : cdic-mcbc@phac-aspc.gc.ca

Indexée dans Index Medicus/MEDLINE SciSearch ${ }^{\circledR}$ et Journal Citation Reports/ Science Edition

Promouvoir et protéger la santé des Canadiens grâce au leadership, aux partenariats, à l'innovation et aux interventions en matière de santé publique - Agence de la santé publique du Canada

Publication autorisée par le ministre de la Santé.

(c) Sa Majesté la Reine du Chef du Canada, représentée par le ministre de la Santé, 2014

ISSN 1925-654X

Pub. 140003

On peut consulter cette publication par voie électronique dans le site Web www.santepublique.gc.ca/mcbc Also available in English under the title: Chronic Diseases and Injuries in Canada 


\title{
Surveillance des maladies chroniques au Canada : Cadre conceptuel d'indicateurs des maladies chroniques
}

\author{
M. T. Betancourt, M.D., M. Sc.; K. C. Roberts, M. Sc.; T-L. Bennett, M. Sc.; E. R. Driscoll, M. Sc.; \\ G. Jayaraman, M.P.H., Ph. D.; L. Pelletier, M.D., M.P.H., FRCPC
}

Cet article a fait l'objet d'une évaluation par les pairs.

\section{Résumé}

Introduction : L'Agence de la santé publique du Canada a conçu le Cadre conceptuel d'indicateurs des maladies chroniques (le Cadre) dans le but de systématiser et d'améliorer la surveillance des maladies chroniques au Canada en instaurant les fondements d'une information uniforme et fiable sur les maladies chroniques et leurs déterminants.

Méthodologie : Des indicateurs de santé nationaux et internationaux, des cadres conceptuels ainsi que des bases de données sur la santé nationale ont été examinés pour identifier les indicateurs potentiels. Afin d'obtenir un ensemble complet et équilibré d'indicateurs pertinents en matière de prévention des maladies chroniques, nous avons élaboré un modèle conceptuel comprenant des « champs de référence » pour le regroupement des indicateurs. Plusieurs critères de sélection ont été appliqués pour le choix des mesures clés. Des consultations approfondies avec un large éventail de partenaires du gouvernement, d'organismes non gouvernementaux et de professionnels de la santé publique ont été réalisées afin d' arriver à un consensus et de perfectionner et valider le Cadre.

Résultats : Le Cadre comprend 41 indicateurs structurés autour de 6 champs de référence : les déterminants sociaux et environnementaux, les facteurs de risque et de protection en bas âge, les facteurs de risque et de protection comportementaux, les conditions à risque, les pratiques de prévention des maladies, l'état de santé global et les impacts sur la santé. Nous avons aussi prévu une mise à jour annuelle des données touchant l'ensemble des indicateurs proposés, que ce soit les estimations nationales, les ventilations par variables démographiques et socioéconomiques ou les tendances temporelles.

Conclusion : Comprendre les données probantes liées aux maladies chroniques et leurs déterminants est nécessaire pour interpréter les tendances et crucial pour élaborer des interventions efficaces en matière de santé publique. Le Cadre et ses produits connexes sont susceptibles de devenir un outil indispensable d'aide à la décision axée sur des données probantes au Canada.

\section{Introduction}

Les maladies chroniques, que ce soit le diabète, le cancer, l'arthrite, les maladies mentales ou les maladies cardiovasculaires et respiratoires chroniques, contribuent grandement à réduire la qualité de vie et à augmenter la perte de productivité, les coûts associés aux hospitalisations et aux soins de santé ainsi que les décès prématurés au Canada ${ }^{1}$. Sur
5 Canadiens âgés de 20 ans et plus, 3 souffrent d'une maladie chronique et 4 risquent de développer une condition chronique ${ }^{2}$.

On sait depuis longtemps que cibler les facteurs de risque et les déterminants des maladies chroniques et s'y attaquer est au fondement de la prévention des maladies chroniques $^{3}$. Comprendre les données probantes portant sur les maladies chroniques et leurs déterminants est donc nécessaire pour interpréter les tendances et crucial pour élaborer des interventions en matière de santé publique pouvant réduire efficacement les taux de maladies chroniques et améliorer la santé et la qualité de vie de la population.

Depuis ses débuts en 2005, l'Agence de la santé publique du Canada (l'Agence) a collaboré avec les ministères de la Santé provinciaux et territoriaux afin de concevoir et de mettre en œuvre plusieurs stratégies, politiques et programmes pancanadiens en matière de maladies chroniques visant à réduire et à prévenir les maladies chroniques. Les nombreux facteurs de risque et de protection associés aux maladies chroniques qui agissent au cours de la vie sont au centre de ces stratégies, notamment de la Stratégie pancanadienne intégrée en matière de modes de vie sains de $2005^{4}$, adoptée par les ministres fédéraux, provinciaux et territoriaux de la Santé, de la Promotion de la santé et du Mode de vie sain du Canada.

Prenant en compte la nécessité d'avoir des données probantes pour éclairer la prise de décisions stratégiques relatives aux politiques et aux programmes ainsi que la volonté de progresser vers l'adoption d'une approche intégrée en matière de prévention des maladies chroniques, l'Agence a jugé nécessaire d'adopter une approche globale en ce qui a trait à la surveillance et à la publication de rapports portant sur les maladies chroniques et leurs déterminants connexes.

Une analyse approfondie de la situation a révélé qu'il n’y avait aucun ensemble 
d'indicateurs national approprié (ou cadre d'indicateurs) capable de répondre aux besoins de l'Agence. Certains cadres, comme le Cadre conceptuel des indicateurs de santé élaboré conjointement par l'Institut canadien d'information sur la santé (ICIS) et Statistique Canada ${ }^{5}$, mettent l'accent sur des champs dépassant le cadre de la surveillance des maladies chroniques (p. ex., une partie du Cadre conceptuel des indicateurs de santé est consacrée à la « performance du système de santé » et aux " caractéristiques de la collectivité et du système de santé », ce qui englobe des champs plus vastes que celui de la surveillance des maladies chroniques). D'autres se focalisent uniquement sur une maladie particulière ou sur une étape de la vie ${ }^{6-8}$. L'Agence a donc entrepris d'élaborer un nouveau cadre conceptuel d'indicateurs axé sur la surveillance intégrée des maladies chroniques.

Ce rapport décrit l'approche adoptée pour l'élaboration du Cadre conceptuel d'indicateurs des maladies chroniques (le Cadre). On a d'abord déterminé les principes directeurs de ce Cadre et conçu un modèle permettant de regrouper les indicateurs afin que sa portée finale réponde aux besoins de l'Agence. On a ensuite organisé de vastes consultations avec des spécialistes du sujet et des scientifiques ainsi qu'avec des décideurs en matière de politiques et de programmes, qui forment le principal public cible du Cadre.

Ce rapport présente aussi les applications actuelles et potentielles du Cadre à la prise de mesures de santé publique efficaces pour la prévention les maladies chroniques au Canada.

\section{Détermination du public cible du Cadre}

La conception du Cadre reposait sur les besoins des décideurs d'avoir accès à des renseignements pertinents et facilement accessibles concernant les tendances liées aux maladies chroniques et qui soient utilisables dans les cas suivants :

- pour déterminer les axes prioritaires en matière de mesures préventives à partir desquels élaborer des politiques et des stratégies en santé;
- pour augmenter les connaissances de la population et des intervenants relativement à la santé et aux facteurs qui l'influencent.

On a ciblé comme principaux utilisateurs des données générées grâce au Cadre les analystes des politiques de santé publique et les praticiens concernés par la prévention des maladies chroniques aux échelles fédérale, provinciale et territoriale. Comprendre les besoins en information de ce public cible était de ce fait essentiel pour concevoir le Cadre et sélectionner des indicateurs pertinents. Ces indicateurs sont aussi utilisables par d'autres programmes et administrations, notamment pour établir des comparaisons.

\section{Principes directeurs}

Nous avons établi quatre principes directeurs touchant la prévention des maladies chroniques et mettant l'accent sur la nécessité de s'attaquer plus efficacement aux facteurs de risque les plus fréquents à l'origine des maladies chroniques et au fardeau que ces maladies représentent au sein des différentes populations. Ces principes ont pour but de guider à la fois la sélection des indicateurs et les rapports les utilisant.

\section{Approche basée sur le parcours de vie}

Les professionnels de la santé affirment que les maladies chroniques et les facteurs de risque touchent les Canadiens de plus en plus jeunes, ce qui confirme l'importance de sélectionner des indicateurs capables de faire ressortir cette tendance émergente ${ }^{9-11}$. C'est pour cette raison que le Cadre comprend des indicateurs ciblés sur les différentes étapes de la vie, de la vie intra-utérine à la fin de vie en passant par l'enfance et l'adolescence (voir figure 1) ${ }^{12,13}$.

\section{Prévention des maladies}

Étant donné son intérêt marqué pour la prévention, l'Agence avait besoin d'aller audelà des rapports concernant des maladies spécifiques pour adopter une approche plus globale tenant compte des déterminants plus généraux de la santé ainsi que des facteurs de risque et des marqueurs biologiques qui contribuent au développement de diverses conditions chroniques.

\section{Approche fondée sur l'équité en santé}

Le fardeau que constituent les maladies chroniques n'est pas réparti uniformément au sein de la population et certains groupes de Canadiens présentent des taux plus élevés de maladies chroniques et bénéficient d'une moins bonne santé. En général, les personnes dont la situation socioéconomique est plus difficile (revenu plus faible, mauvaises conditions de travail, faible soutien social, etc.) sont en moins bonne santé que celles jouissant d'un statut socioéconomique plus élevé. La compréhension des différences relatives à la santé entre ces groupes de population est cruciale pour concevoir des programmes et des politiques qui permettent de réduire ces différences.

Afin de connaître ces disparités au sein de la population, nous avons sélectionné en priorité pour le Cadre des indicateurs stratifiables conformément aux recommandations de l'Organisation mondiale de la santé relatives à l'élaboration d'un " système national de surveillance de l'équité en santé ${ }^{14}$.

\section{Multimorbidité}

Le fardeau associé aux maladies chroniques n'est pas simplement la somme des conséquences de chaque maladie chronique. Souvent, il implique une interaction complexe entre plusieurs maladies, ce qui entraîne des problèmes supplémentaires pour les Canadiens. Ce concept de multimorbidité ${ }^{15}$ est un enjeu de santé publique crucial et il permet de prédire de manière indépendante certains effets néfastes sur la santé, dont la diminution de la qualité de vie et l'augmentation des coûts liés aux soins de santé, de l'incapacité et du décès prématuré. C'est pour cette raison que l'Agence a fait des indicateurs pertinents de multimorbidité une composante clé du Cadre.

\section{Structurer le Cadre}

Nous sommes loin de la pénurie de données nationales ou internationales sur 
FIGURE 1

Diagramme illustrant le parcours de vie

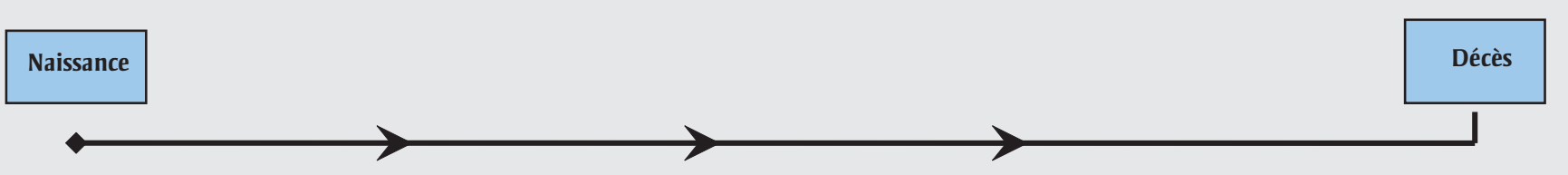

Facteurs socioéconomiques (éducation, revenu, emploi, soutien social)

Facteurs environnementaux (environnement bâti, exposition à des contaminants environnementaux)

Facteurs prénataux, au début de la vie et pendant

I'enfance (faible poids à la naissance, allaitement

maternel, exposition à la fumée secondaire)

Facteurs de risque comportementaux (unique $\rightarrow$ concentration de facteurs de risque)

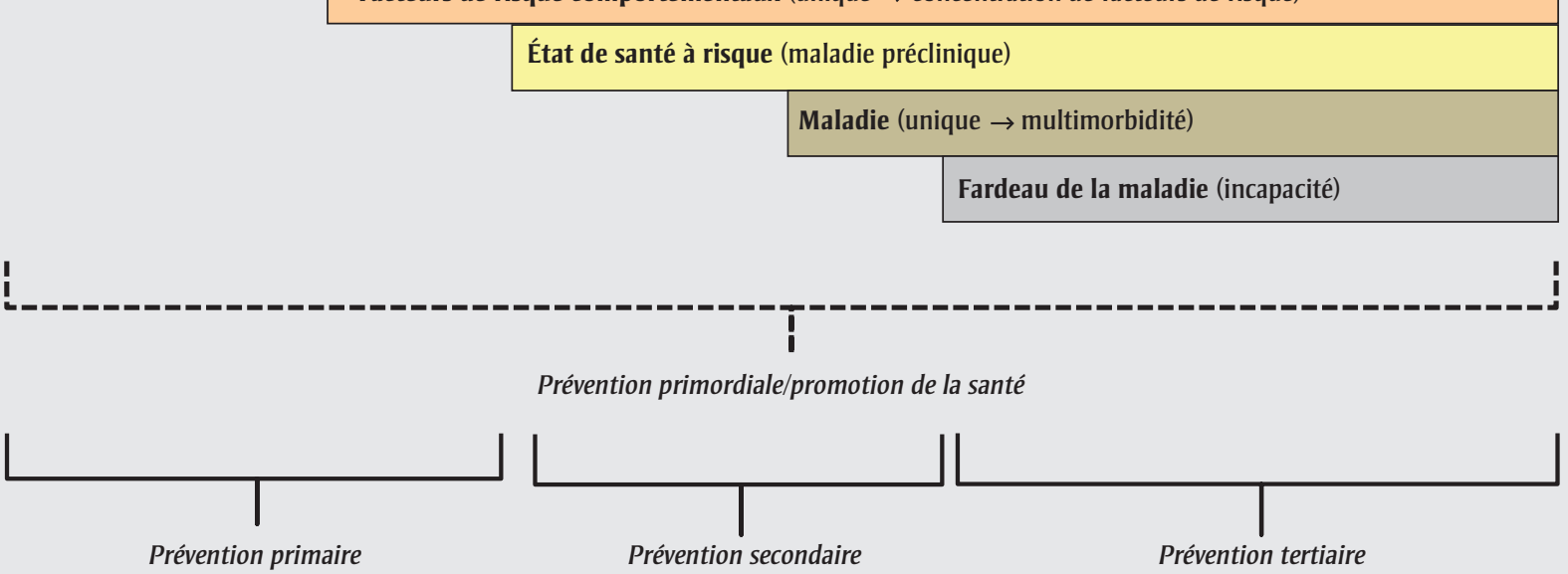

la santé utilisables pour produire des rapports sur une vaste gamme de champs relatifs à la santé des Canadiens, que ces données soient recueillies en utilisant des méthodes actives (comme des études) ou passives (comme l'utilisation secondaire de données administratives). Le défi dans la conception de ce Cadre résidait plutôt dans le choix des mesures les plus importantes et les plus pertinentes pour dresser un portrait fidèle des maladies chroniques au Canada tout en demeurant en harmonie avec d'autres cadres conceptuels connexes.

Pour être sûre d'obtenir un ensemble complet et équilibré d'indicateurs pertinents et importants pour la prévention des maladies chroniques, l'Agence a défini au préalable ses critères de sélection des indicateurs (tableau 1), a élaboré un modèle conceptuel de champs de référence facilitant leur regroupement (tableau 2) et a mis au point un processus de priorisation.

\section{Critères de sélection des indicateurs}

Les critères de sélection des indicateurs sont conformes aux recommandations de la littérature et ont déjà été utilisés par plusieurs cadres conceptuels d'indicateurs de santé nationaux et internationaux ${ }^{16-19}$. Ils sont présentés dans le tableau 1. Dans la mesure du possible, on a tenu compte à chaque étape de la sélection de l'harmonie avec les cadres existants et avec les indicateurs déjà utilisés sur une base régulière.

\section{Champs de référence du modèle conceptuel}

L'Agence a retenu six champs de référence (voir tableau 2) permettant de regrouper les indicateurs sélectionnés. Pour garantir un ensemble d'indicateurs équilibré entre les champs, chacun d'entre eux devait inclure au moins trois indicateurs.

\section{Alimenter le cadre}

Une analyse approfondie des rapports publiés et de la littérature grise rédigée par l'Agence et par d'autres organismes canadiens $^{20-25}$ et internationaux ${ }^{26-30}$ sur le sujet ainsi qu'un examen des principales bases de données nationales sur la santé (dont le Système national de surveillance des maladies chroniques et l'Enquête sur la santé dans les collectivités canadiennes, entre autres) ont permis de présélectionner 283 mesures d'indicateurs susceptibles de relever des champs de référence du Cadre. Deux examinateurs indépendants connaissant bien le Système national de surveillance des maladies chroniques ont sélectionné 
TABLEAU 1

Critères de sélection des indicateurs

\begin{tabular}{|c|c|}
\hline Critères & Description \\
\hline Pertinence & $\begin{array}{l}\text { L'indicateur est incontestablement pertinent pour la prévention et le contrôle des maladies chroniques, ou bien il pourrait } \\
\text { servir d'indicateur de substitution (norme de référence) pour la mesure qui le sous-tend. }\end{array}$ \\
\hline Exactitude & $\begin{array}{l}\text { Intégrité scientifique : Les preuves scientifiques qui établissent le lien entre la performance de l'indicateur et les maladies chroniques } \\
\text { sont solides. } \\
\text { Validité : L'indicateur semble offrir une mesure raisonnable de ce qu'il est censé mesurer (validité apparente) et les composantes } \\
\text { de l'indicateur sont sensées (validité conceptuelle). } \\
\text { Fiabilité : On obtiendrait les mêmes résultats si on répétait les mesures dans des conditions identiques. }\end{array}$ \\
\hline Incitation à l'action & $\begin{array}{l}\text { Offre une information qui peut inciter à agir en vue d'un changement : éclaire et influence les décisions politiques ou les } \\
\text { financements, modifie le comportement des prestataires de services de santé ou augómente la compréhension générale au } \\
\text { sein de la collectivité (p. ex. améliore les comportements, les résultats ou encore l'utilisation des services de santé). }\end{array}$ \\
\hline Faisabilité & $\begin{array}{l}\text { Les données disponibles sont suffisamment de bonne qualité, ou bien une collecte de données peut être mise en place à } \\
\text { un coût relativement faible. }\end{array}$ \\
\hline
\end{tabular}

un sous-ensemble de 130 mesures d'indicateurs en se fondant sur 2 des 6 critères de sélection, à savoir la pertinence en ce qui a trait aux maladies chroniques et l'incitation potentielle à l'action. Les 130 mesures d'indicateurs ont ensuite été ramenées à 45 indicateurs en utilisant les résultats d'une équipe d'experts en surveillance des maladies chroniques de l'Agence (professionnels de la santé publique, épidémiologistes et biostatisticiens) par le biais d'un processus Delphi modifié (c.-à-d. que les barèmes de classement fondés sur les 6 critères de sélection et les discussions ouvertes ont été utilisés itérativement pour en arriver à un consensus).
Pour s'assurer que le Cadre ciblait des mesures et des indicateurs globaux, fondés sur des données probantes, significatifs et pertinents pour les concepteurs de programmes et les décideurs à l'échelle fédérale, plusieurs groupes de l'Agence et de Santé Canada ont été consultés relativement à ce choix de 45 indicateurs. À la suite des commentaires reçus, 3 indicateurs supplémentaires ont été intégrées au Cadre. Cette liste révisée de 48 indicateurs a ensuite été transmise à un large éventail de partenaires gouvernementaux, d'organismes non gouvernementaux et de professionnels de la santé publique (75 personnes relevant de 7 organismes ainsi que quelques réponses anonymes) pour qu'elle soit examinée en fonction des critères de sélection de départ (tableau 1). À la suite des commentaires reçus, 2 mesures supplémentaires ont été ajoutées au Cadre et 11 ont été supprimées, ce qui a conduit à un total de 39 indicateurs.

Les mesures suggérées au cours des deux rondes de consultations qui ne répondaient pas aux 6 critères de sélection de départ ont été inscrites dans une liste pour consultation ultérieure (p. ex. qualité de l'air, indice de défavorisation sociale, indice de santé mentale, troubles du sommeil).

Il est important de noter que l'ensemble du processus de sélection des indicateurs

\section{TABLEAU 2}

\section{Champs de référence et explication justificative}

\begin{tabular}{|c|c|}
\hline Champ & Explication justificative \\
\hline $\begin{array}{l}\text { Déterminants sociaux } \\
\text { et environnementaux }\end{array}$ & Fournit de l'information sur les facteurs contextuels et les mesures d'équité qui influencent la santé. \\
\hline $\begin{array}{l}\text { Facteurs de risque et de } \\
\text { protection en bas âge }\end{array}$ & $\begin{array}{l}\text { Fournit de l'information sur les premiers facteurs de risque et de protection connus pour leur influence sur l'état de santé } \\
\text { tout au long de la vie et pouvant être ciblés dans le cadre d'activités de prévention primordiale }{ }^{a} \text { et primaire }{ }^{b} \text {. }\end{array}$ \\
\hline $\begin{array}{l}\text { Facteurs de risque et de } \\
\text { protection comportementaux }\end{array}$ & $\begin{array}{l}\text { Fournit de l'information sur les facteurs de risque et de protection comportementaux individuels agissant sur la } \\
\text { probabilité de développer des maladies chroniques et pouvant être ciblés grâce à la prévention primordiale et primaire. }\end{array}$ \\
\hline Conditions à risque & $\begin{array}{l}\text { Fournit de l'information sur les facteurs de risque intermédiaires associés à la maladie chronique pouvant être ciblés } \\
\text { grâce à la prévention secondaire }{ }^{c} \text {. }\end{array}$ \\
\hline $\begin{array}{l}\text { Pratiques de prévention } \\
\text { des maladies }\end{array}$ & $\begin{array}{l}\text { Fournit de l'information sur les pratiques de dépistage et de prévention des maladies utilisées pour la prévention } \\
\text { secondaire ou tertiaire }^{\mathrm{d}} \text { des maladies chroniques. }\end{array}$ \\
\hline $\begin{array}{l}\text { État de santé global et } \\
\text { impacts sur la santé }\end{array}$ & $\begin{array}{l}\text { Fournit de l'information sur l'ampleur et les conséquences des maladies chroniques et de la multimorbidité ainsi que } \\
\text { sur l'impact de ces conséquences sur la qualité de vie, l'incapacité et le décès prématuré. }\end{array}$ \\
\hline
\end{tabular}


était itératif. Ainsi, à chaque étape de choix des indicateurs, on a examiné les versions les plus récentes de certains cadres d'indicateurs et d'indicateurs de la santé nationaux et internationaux pour s'assurer que le Cadre était à la fois complet et en harmonie avec les autres cadres. Par exemple, les mesures du tabagisme correspondent aux indicateurs du tabagisme de Santé Canada. Le processus d'analyse et les limites des données ont aussi justifié l'ajout de 2 nouvelles mesures (une mesure objective de l'activité physique chez les enfants et les jeunes et une mesure approximative de la mortalité associée au diabète, soit le taux de mortalité toutes causes confondues chez les personnes atteintes du diabète comparé à celui des personnes non atteintes du diabète). La liste finale des indicateurs du Cadre (tableau 3) a été perfectionnée après consultations poussées auprès de notre public cible, en utilisant également un processus itératif. Sur les 41 indicateurs, 36 peuvent faire dès à présent l'objet d'un rapport et 5 - soutien social, milieu physique, syndrome métabolique, combinaison de facteurs de risque et dépistage de l'hypertension artérielle — sont toujours en développement (voir annexe A). Pour une description détaillée de chaque indicateur voir Annexe B.

La figure 2 offre un diagramme explicatif du processus de sélection des indicateurs.

\section{Rapports fondés sur des indicateurs utilisant le Cadre}

L'un des principaux objectifs du Cadre est de garantir que l'Agence et les autres intervenants clés publient des rapports statistiques uniformes. L'accès à l'information est pour cela essentiel, et le type et la portée des publications ont aussi une incidence. On prévoit en lien avec cet objectif une mise à jour annuelle incluant les données les plus récentes pour chaque indicateur du Cadre. Étant données les variations dans la fréquence de collecte des données entre les différentes sources, tous les indicateurs ne seront pas mis à jour chaque année, mais le but est de produire néanmoins annuellement une publication sur l'ensemble des indicateurs de base proposés, ce qui fournira une source de renseignements globale, régu- lière et accessible au public. Ce processus permettra certainement une meilleure interprétation commune de l'état des maladies chroniques, de leurs déterminants et des lacunes en matière de connaissances à ce sujet, ce qui entraînera sûrement en retour des partenariats stratégiques axés sur les données probantes et des investissements dans des programmes de réduction des effets néfastes sur la santé et des disparités en matière de santé.

Outre la publication régulière de la liste d'indicateurs de base, nous prévoyons des publications thématiques ciblant les problèmes et tendances en émergence, approfondissant les 6 champs de référence et leurs interactions et analysant des thèmes transversaux comme la multimorbidité, la combinaison des facteurs de risque, les inégalités liées à la santé ou certaines populations comme celle des enfants et des jeunes.

\section{Applications du Cadre à la prise de mesures en santé publique efficaces}

L'Agence s'est engagée à utiliser le Cadre pour produire des publications sur les indicateurs de base liés aux maladies chroniques et leurs déterminants connexes. Plusieurs provinces et territoires du Canada ont aussi fait part de l'intérêt qu'ils portaient au Cadre pour la collecte de données visant à mesurer des performances, ou pour répondre aux processus de planification stratégique, entre autres. L'Alliance canadienne de surveillance régionale des facteurs de risque, un réseau pancanadien de professionnels de la santé publique, facilite les travaux collaboratifs entre provinces et territoires. À l'échelle internationale, en tant que centre de collaboration de l'Organisation mondiale de la santé en matière de politiques relatives aux maladies non transmissibles, le Centre de prévention et de contrôle des maladies chroniques de l'Agence utilise déjà le Cadre pour faire un choix éclairé des indicateurs qui vont être utilisés pour surveiller et mesurer les maladies chroniques et leurs déterminants à l'échelle mondiale. Par exemple, le Cadre a déjà été utilisé pour informer la finalisation du «Cadre mondial de suivi pour les maladies non transmissibles ${ }^{30}$ de l'Organisation mondiale de la santé ainsi que pour déterminer et prioriser quelques uns des principaux indicateurs " Les indicateurs et les cibles en matière de maladies non transmissibles » de l'Organisation panaméricaine de la santé, qui servent au suivi des taux de mortalité et à l'évaluation des progrès réalisés dans la prévention et le contrôle des maladies non transmissibles, respectivement à l'échelle internationale et dans les Amériques. Plus la production de publications utilisant le Cadre sera importante, plus nous aurons des rapports uniformes sur un ensemble d'indicateurs de base permettant d'établir des comparaisons sur les tendances temporelles et entre provinces et territoires.

Étant donné que le processus d'élaboration du Cadre a permis de relever certaines lacunes dans les indicateurs, l'Agence collabore avec Statistique Canada et d'autres intervenants pour intégrer des éléments découlant d'enquêtes sur la santé auprès de la population nationale et pouvant constituer une source importante de données en matière de surveillance des maladies chroniques. Outre les enquêtes, on pourrait concevoir des outils de collecte de données novateurs pour fournir des mesures objectives dans de nouveaux champs ou des champs présentant des lacunes. La nécessité de demeurer informé des nouvelles données probantes pour créer de nouveaux indicateurs ou adapter ceux qui existent va exiger l'établissement de partenariats novateurs et la participation active des chercheurs. Cela permettra en même temps de renforcer la contribution du Canada à la recherche sur les manières d'aborder la question de la prévention des maladies chroniques.

\section{Limites}

L'ensemble d'indicateurs proposé pour le Cadre comporte certaines limites. D’abord, la portée de la sélection des indicateurs a été limitée par la disponibilité des données nationales et par l'obtention restreinte de données stratifiables selon certaines variables socioéconomiques. Deuxièmement, certains indicateurs ne peuvent pas encore faire l'objet de rapports ciblant certains sous-groupes de population (p. ex. populations autochtones, immigrants) et les enquêtes 
TABLEAU 3

Version finale du Cadre d'indicateurs

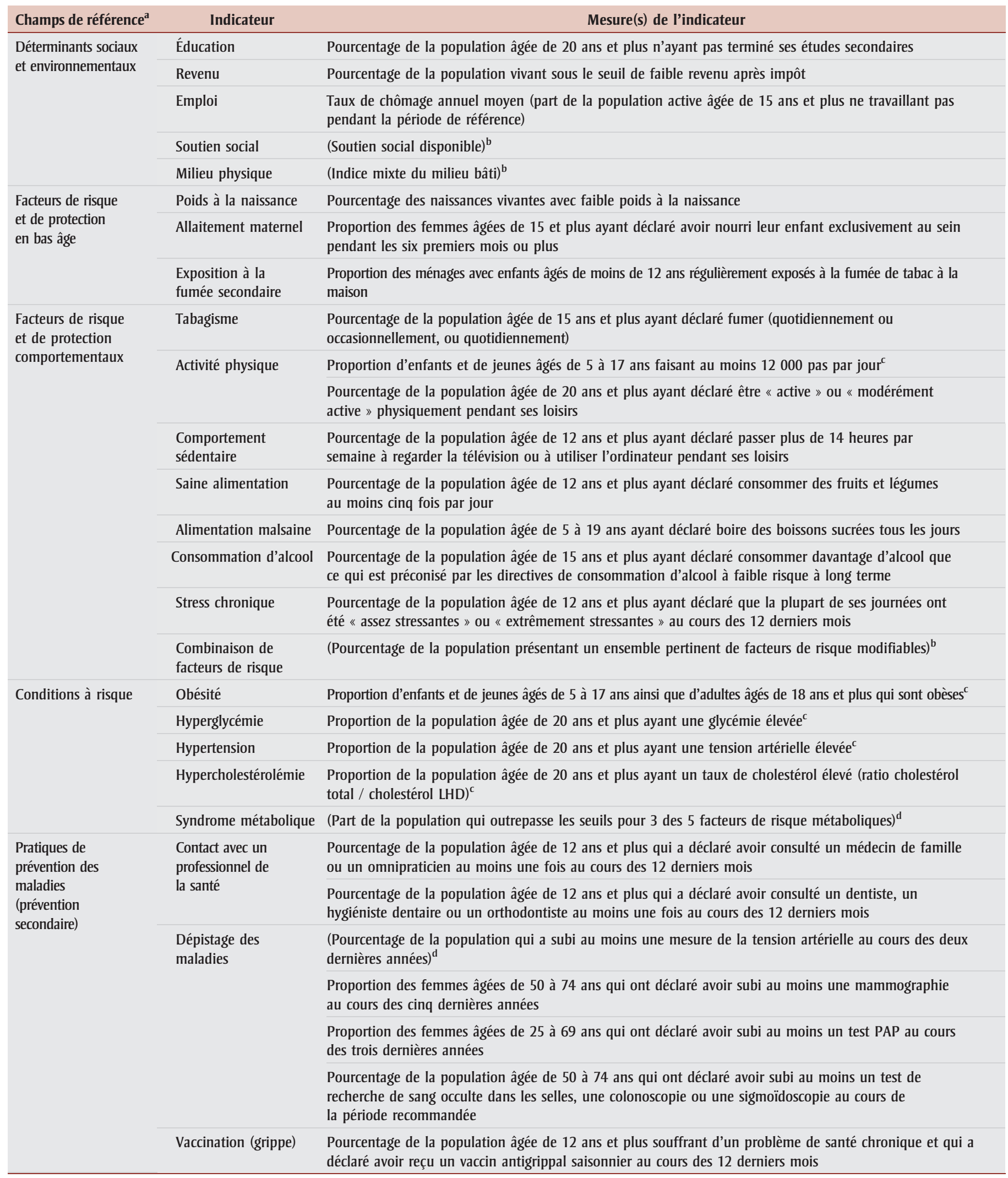

Suite page suivante 
TABLEAU 3 (Suite)

Version finale du Cadre d'indicateurs

\begin{tabular}{|c|c|c|}
\hline Champs de référence $^{\mathrm{a}}$ & Indicateur & Mesure(s) de l'indicateur \\
\hline \multirow{14}{*}{$\begin{array}{l}\text { État de santé global } \\
\text { et impacts sur la santé }\end{array}$} & \multirow[t]{4}{*}{ Santé générale } & Pourcentage de la population âgée de 12 ans et plus ayant qualifié sa santé de « très bonne » ou « excellente » \\
\hline & & Pourcentage de la population âgée de 12 ans et plus ayant qualifié sa santé mentale de « très bonne » ou « excellente » \\
\hline & & Espérance de vie (à la naissance, à 65 ans) \\
\hline & & Espérance de vie ajustée en fonction de l'état de santé (à la naissance, à 65 ans) \\
\hline & \multirow[t]{3}{*}{ Morbidité } & $\begin{array}{l}\text { Prévalence des maladies chroniques majeures : cancer, diabète, maladies cardiovasculaires, maladie } \\
\text { pulmonaire obstructive chronique }\end{array}$ \\
\hline & & $\begin{array}{l}\text { Prévalence de maladies chroniques spécifiques : diabète, maladies cardiovasculaires, cancers, maladies } \\
\text { respiratoires chroniques, maladies musculosquelettiques, maladies mentales, maladies neurodégénératives (MAAD) }\end{array}$ \\
\hline & & $\begin{array}{l}\text { Taux d'incidence de maladies chroniques spécifiques : diabète, maladies cardiovasculaires, cancers, } \\
\text { maladies respiratoires chroniques }\end{array}$ \\
\hline & Multimorbidité & Pourcentage de la population âgée de 20 ans et plus ayant de multiples maladies chroniques \\
\hline & Incapacité & $\begin{array}{l}\text { Part de la population âgée de } 12 \text { ans et plus ayant déclaré être limitée " parfois » ou « souvent » dans ses } \\
\text { activités en raison de problèmes de santé ou de maladie }\end{array}$ \\
\hline & \multirow[t]{5}{*}{ Mortalité } & $\begin{array}{l}\text { Taux de mortalité attribuable à une maladie chronique importante : maladies cardiovasculaires, cancer, } \\
\text { maladies respiratoires chroniques }\end{array}$ \\
\hline & & $\begin{array}{l}\text { Taux de mortalité attribuable à des maladies chroniques spécifiques (maladies cardiovasculaires, } \\
\text { cancer, maladies respiratoires chroniques) ou au suicide }\end{array}$ \\
\hline & & $\begin{array}{l}\text { Ratio du taux de mortalité toutes causes confondues chez les personnes atteintes de diabète sur } \\
\text { celui des personnes non atteintes de diabète }\end{array}$ \\
\hline & & $\begin{array}{l}\text { Années potentielles de vie perdues selon la cause du décès : cancer, maladies cardiovasculaires, } \\
\text { maladies respiratoires chroniques, suicide }\end{array}$ \\
\hline & & $\begin{array}{l}\text { Probabilité inconditionnelle de mourir (\%) entre } 30 \text { et } 69 \text { ans de l'une ou plusieurs des principales } \\
\text { maladies chroniques : maladies cardiovasculaires, cancer, maladies respiratoires chroniques, diabète }\end{array}$ \\
\hline
\end{tabular}

Abréviations : Cholestérol LHD, cholestérol à lipoprotéines de haute densité; MAAD, maladie d'Alzheimer et autres démences.

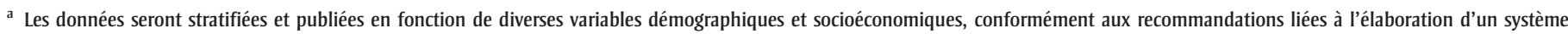
national de surveillance de l'équité en santé ${ }^{14}$ : groupes d'âge, sexe, au moins 2 marqueurs sociaux (p. ex. éducation, revenu, ethnicité, statut d'immigrant), au moins un marqueur régional (province/territoire, milieu rural/urbain) et le statut d'Autochtone dans la mesure du possible.

b Ces mesures sont toujours en cours d'élaboration, analysées au même titre que d'autres lacunes statistiques.

c Ces mesures sont fondées sur des données réellement mesurées.

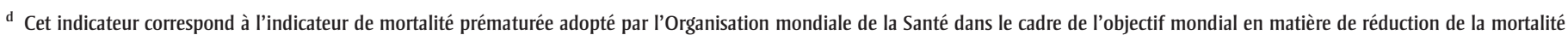
prématurée due à des maladies non transmissibles ${ }^{31}$.

nationales actuelles ne prennent pas correctement en compte certaines populations clés (p. ex. enfants de moins de cinq ans). Par conséquent, la priorité est de lancer des travaux additionnels visant à favoriser la collecte de données en étendant la portée des enquêtes actuelles sur la santé de la population ou en utilisant des instruments d'enquête novateurs.

Troisièmement, compte tenu de la complexité des facteurs proximaux et distaux contribuant aux maladies chroniques, il demeure essentiel d'analyser les nouvelles données probantes confirmant les influences sociétales, environnementales et communautaires les concernant. Il est important de souligner que certains indicateurs potentiellement utiles (p. ex. la qualité de l'air, l'indice de défavorisation sociale, l'indice de santé mentale, les troubles du sommeil) n'ont pas été inclus dans le Cadre parce qu'ils ne répondaient pas à l'un des critères de sélection, celui de la faisabilité de la collecte de données. Enfin, la sélection des indicateurs de protection et de résilience demeure faible, et il s'agit là d'un domaine qui nécessite un engagement actif. Le défi va consister, pour que la liste d'indicateurs demeure limitée et gérable, à maintenir un équilibre entre le caractère inclusif et flexible du Cadre d'un côté et l'obligation d'assurer une production continue de publications sur l'ensemble des indicateurs de base pour les analyses des tendances de l'autre.

\section{Conclusion}

L'Agence de la santé publique du Canada a entrepris de concevoir ce Cadre conceptuel d'indicateurs des maladies chroniques pour permettre la publication régulière de rapports sur l'état des maladies chroniques au Canada. Malgré certaines limites, l'approche structurée et itérative utilisée pour sa conception assure au Cadre et à ses produits afférents le potentiel d'un outil indispensable à la prise de décision éclairée par des données probantes au Canada. 
FIGURE 2

Diagramme du processus de sélection des indicateurs

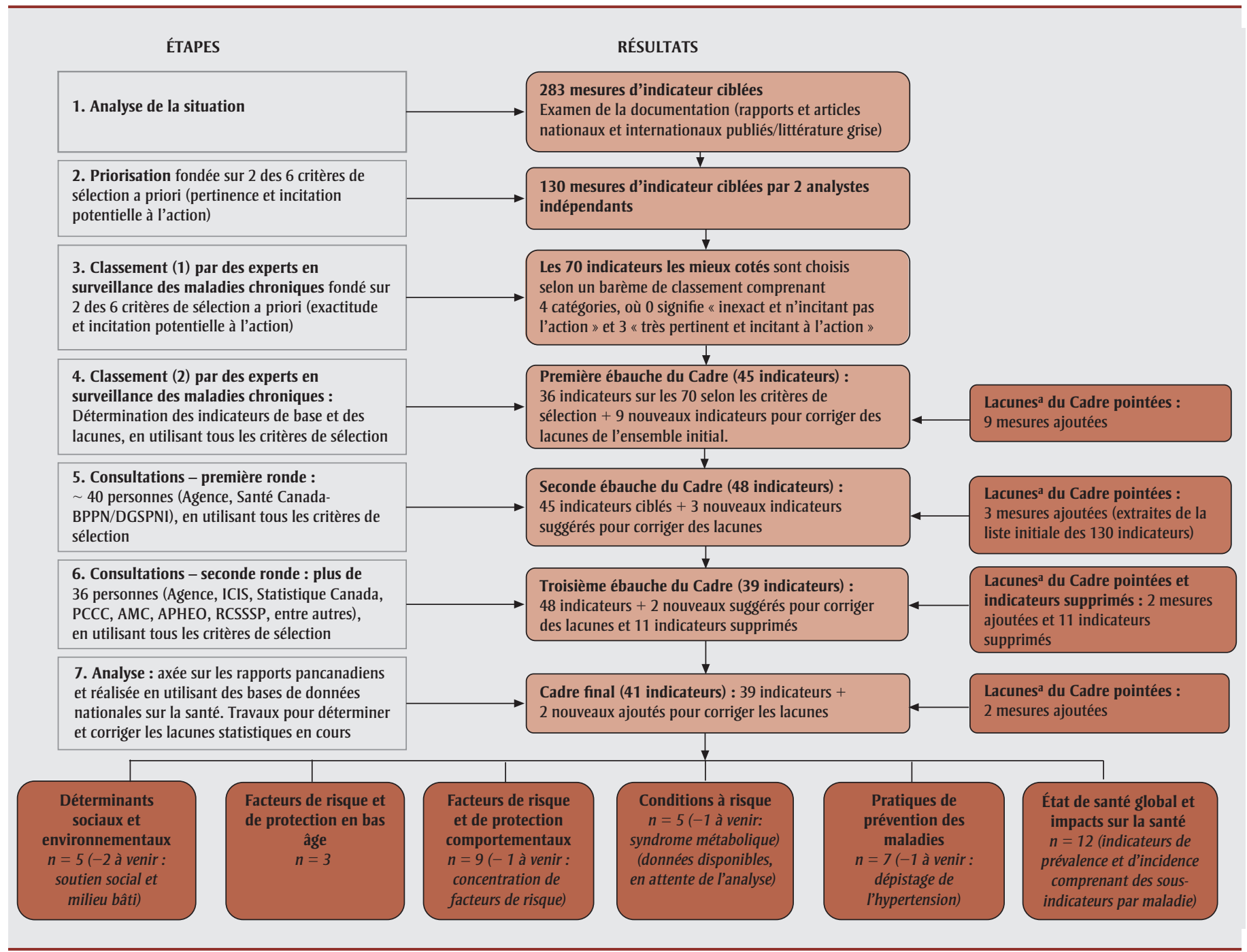

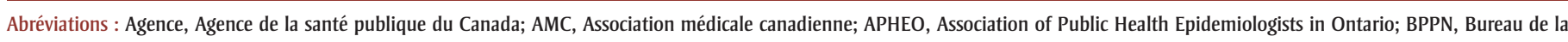

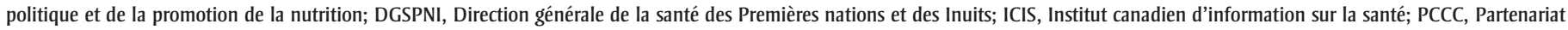
canadien contre le cancer; RCSSSP, Réseau canadien de surveillance sentinelle en soins primaires.

${ }^{a}$ Lacunes du Cadre : principales mesures considérées comme manquantes selon les commentaires reçus pendant les consultations et le processus d'analyse statistique (étapes 4 à 7 ).

\section{Références}

1. Comité consultatif sur la santé de la population et la sécurité de la santé, Groupe de travail sur les systèmes de surveillance des facteurs de risque reliés aux maladies chroniques. Accroître la capacité de surveillance des facteurs de risque et des déterminants reliés. Ottawa (Ont.) : Groupe de travail sur les systèmes de surveillance des facteurs de risque reliés aux maladies chroniques; 2005.
2. Agence de la santé publique du Canada. Feuillet de renseignements: Initiatives du gouvernement du Canada en matière de lutte contre les maladies chroniques [Internet]. Ottawa (Ont.) : [mis à jour le 19 sept. 2011; consultation le 24 juin 2013]. Consultable à la page : http://www.phacaspc.gc.ca

/media/nr-rp/2011/2011_0919-fs-fr-fra.php

3. Lalonde M. Nouvelle perspective de la santé des Canadiens. Ottawa (Ont.) : Ministère de la Santé nationale et du Bienêtre social; 1974.
4. Réseau intersectoriel de promotion des modes de vie sains; Groupe de travail sur les modes de vie sains; Comité consultatif fédéral-provincial-territorial sur la santé de la population et de la sécurité de la santé. Stratégie pancanadienne intégrée en matière de modes de vie sains. Ottawa (Ont.) : Ministre de la Santé; 2005.

5. Statistique Canada. Cadre conceptuel des indicateurs de santé [Internet]. Ottawa (Ont.) : Statistique Canada; [mis à jour le 12 déc. 2013; consultation le 6 jan. 2014]. Consultable en ligne à la page : http:// www.statcan.gc.ca/pub/82-221-x/2013001 /hifw-fra.htm 
6. LeMessurier J, O’Donnell S, Walsh P, McRae L, Bancej C. L'Élaboration d'indicateurs nationaux pour la surveillance de l'ostéoporose au Canada. Maladies chroniques et blessures au Canada. 2012;32(2):114-121.

7. Agence de la santé publique du Canada. Programmes organisés de dépistage du cancer du sein au Canada: Rapport sur la performance des programmes en 2005 et 2006. Ottawa (Ont.) : Agence de la santé publique du Canada; 2010.

8. Agence de la santé publique du Canada. Rapport sur la santé prénatale au Canada, édition 2008. Ottawa (Ont.) : Agence de la santé publique du Canada; 2008.

9. Amed S, Daneman D, Mahmud FH, Hamilton J. Type 2 diabetes in children and adolescents. Expert Rev Cardiovasc Ther. 2010;8(3):393-406. DOI: 10.1586/erc.10.15

10. Camhi SM, Katzmarzyk PT. Prevalence of cardiometabolic risk factor clustering and body mass index in adolescents. J Pediatr. 2011;159(2):303-7. DOI: 10.1016/j.jpeds .2011 .01 .059

11. Ford ES, Mokdad AH, Ajani UA. Trends in risk factors for cardiovascular disease among children and adolescents in the United States. Pediatrics. 2004;114(6):1534-44.

12. Kuh D, Ben-Shlomo Y. A life course approach to chronic disease epidemiology. $2^{\mathrm{e}}$ éd. London (UK) : Oxford University; 2004.

13. Ben-Shlomo Y, Kuh D. A life course approach to chronic disease epidemiology: conceptual models, empirical challenges and interdisciplinary perspectives. Int $\mathrm{J}$ Epidemiol. 2002;31(2):285-93.

14. Commission des déterminants sociaux de la santé. Combler le fossé en une génération : Instaurer l'équité en santé en agissant sur les déterminants sociaux : Rapport final de la Commission des déterminants sociaux de la santé. Genève $(\mathrm{CH})$ : Organisation mondiale de la santé; 2008.

15. Boyd C, Fortin M. Future of multimorbidity research: how should understanding of multimorbidity inform health system design. Public Health Rev. 2010;32(2):451-74.

16. Flowers J, Hall P, Pencheon D. Public health indicators. Public Health. 2005; 119(4):239-45.
17. Project for an Ontario Women's HealthEvidence Based Report (POWER). POWER Indicators [Internet]. Toronto (Ont.) : St. Michael's Hospital; [consultation le 24 juin 2013]. Consultable en ligne à la page : http:// powerstudy.ca/indicators/power-indicators/

18. Bird SM, Cox D, Farewell VT, Goldstein H, Holt $\mathrm{T}$, Smith PC. Working Party on Performance Monitoring in the Public Service. Performance indicators: good, bad, and ugly. J. R. Statist. Soc. A. 2005;168(1):1-27

19. Pencheon D. The good indicators guide: understanding how to use and choose indicators. [place unknown]: NHS Institute for Innovation and Improvement, Association of Public Health Observatories; 2008.

20. Statistique Canada. Indicateurs de la santé [Internet]. Ottawa (Ont.) : Statistique Canada; [consultation le 24 juin 2013]. Consultable en ligne à la page : http:// www.statcan.gc.ca/pub/82-221-x/2011002 /def/def2-fra.htm

21. Santé Canada. Les Canadiens et les Canadiennes en santé : Rapport fédéral sur les indicateurs comparables de la santé en 2008. Ottawa (Ont.) : Gouvernement du Canada; 2008.

22. Population and Public Health Data Expert Group. Population and public health indicators for British Columbia. Vancouver (BC): Provincial Health Services Authority; 2008.

23. Association of Public Health Epidemiologists in Ontario. Core indicators [Internet]. Ontario: APHEO; [mis à jour en juin 2012; consultation le 24 juin 2013]. Consultable en ligne à la page: http://www.apheo.ca /index.php?pid $=55$

24. Ressources humaines et Développement des compétences Canada. Indicateurs de mieux-être au Canada. Santé - Auto-évaluation de la santé [Internet]. Ottawa (Ont.) : Emploi et Développement social Canada; [consultation le 24 juin 2013]. Consultable en ligne à la page: http://www4.rhdcc.gc.ca /.3nd.3c.1t.4r@-fra.jsp?iid=10
25. Groupe d'experts de la promotion de la santé de la population; Groupe des modes de vie sains. Indicateurs des disparités sur le plan de la santé : Un rapport du Groupe d'experts de la promotion de la santé de la population et du Groupe des modes de vie sains pour le Réseau pancanadien de santé publique. Ottawa (Ont.) : Réseau pancanadien de santé publique; 2009.

26. Public Health Observatories. Health profiles 2011 are comparison charts [Internet]. London (UK) : Public Health England; [consultation le 24 juin 2013]. Consultable en ligne à la page : http://www.apho.org.uk /default.aspx?QN = HP_COMPARISON_RAGS _2011

27. National Center for Chronic Prevention and Health Promotion. Chronic disease indicators [Internet]. Atlanta (GA) : Centers for Disease Control and Prevention; [consultation le 24 juin 2012]. Consultable en ligne à la page : http://apps.nccd.cdc.gov/cdi/

28. Organisation de coopération et de développement économiques (OCDE). État de Santé [Internet]. Paris (FR) : OCDE; [consultation le 24 juin 2013]. Consultable en ligne à la page : http://stats.oecd.org/index.aspx? lang $=$ fr\&SubSessionId $=$ af1b0641-de29-4079 -919a-5ac2f0890fof\&themetreeid $=9$

29. Healthy People 2020. Leading health indicators [Internet]. Washington (DC) : Office of Disease Prevention and Health Promotion; [consultation le 24 juin 2013]. Consultable en ligne à la page : http:// www.healthypeople.gov/2020/LHI/default .aspx

30. Australian Institute of Health and Welfare. Chronic disease indicator database [Internet]. Sydney (AU) : AIHW; [consultation le 24 juin 2013]. Consultable en ligne à la page : http://www.aihw.gov.au/chronic -disease-indicator/

31. Organisation mondiale de la santé. Annexe 1: Cadre global mondial de suivi et cibles mondiales volontaires pour la prévention et la lutte contre les maladies non transmissibles. Genève $(\mathrm{CH})$ : Organisation mondiale de la santé; [21 déc. 2011]. 
ANNEXE A

CADRE D'INDICATEURS DES MALADIES CHRONIQUES, STATISTIQUES RAPIDES, ÉDITION PRINTEMPS 2014

\begin{tabular}{lcc} 
GROUPE & MESURE(S) D'INDICATEUR & DONNÉES LES PLUS \\
D'INDICATEURS & SOURCE DES \\
RENCENTES ${ }^{\mathrm{a}}$ & $\begin{array}{c}\text { DONNÉES } \\
\text { (ANNÉE) }\end{array}$ \\
\hline
\end{tabular}

DÉTERMINANTS SOCIAUX ET ENVIRONNEMENTAUX

\begin{tabular}{llllll}
\hline Éducation & $\%$ de la population âgée de 20 ans et plus n'ayant pas terminé ses études secondaires & 13,4 \% & ESCC (2011-2012) \\
\hline Revenu & $\%$ de l'ensemble de la population vivant sous les seuils de faible revenu après impôt & $8,8 \%$ & EDTR (2011) \\
\hline Emploi & $\begin{array}{l}\text { Taux de chômage annuel moyen (\% de la population active âgée de } 15 \text { ans et plus ne } \\
\text { travaillant pas pendant la période de référence) }\end{array}$ & $7,2 \%$ & EPA (2012)
\end{tabular}

FACTEURS DE RISQUE ET DE PROTECTION EN BAS ÂGE

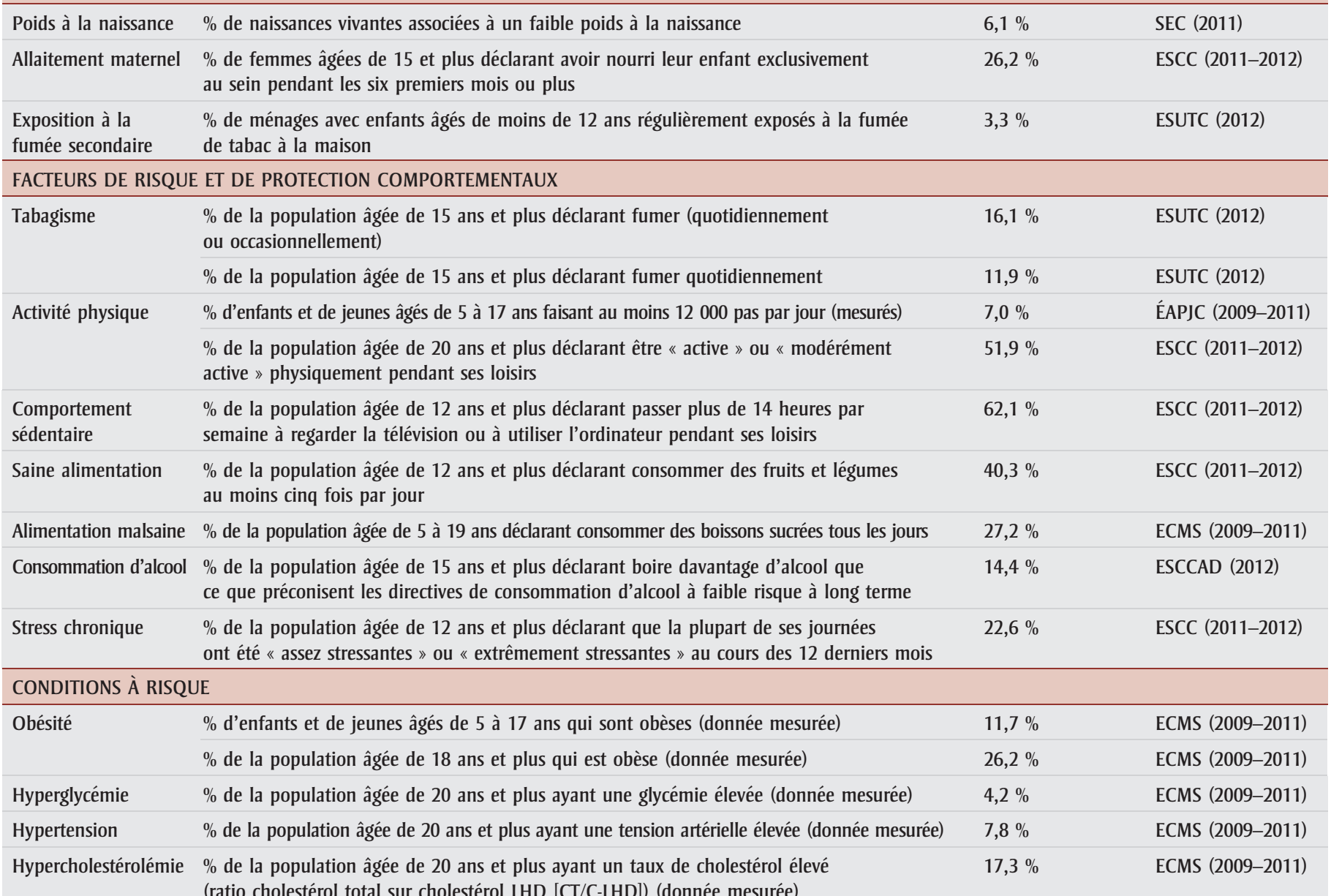

\section{PRATIQUES DE PRÉVENTION DES MALADIES (PRÉVENTION SECONDAIRE)}

\begin{tabular}{|c|c|c|c|}
\hline \multirow{2}{*}{$\begin{array}{l}\text { Contact avec un } \\
\text { professionnel de } \\
\text { la santé }\end{array}$} & $\begin{array}{l}\text { \% de la population âgée de } 12 \text { ans et plus déclarant avoir consulté un médecin de famille } \\
\text { ou un omnipraticien au moins une fois au cours des } 12 \text { derniers mois }\end{array}$ & $75,2 \%$ & ESCC (2012) \\
\hline & $\begin{array}{l}\text { \% de la population âgée de } 12 \text { ans et plus déclarant avoir consulté un dentiste, un } \\
\text { hygiéniste dentaire ou un orthodontiste au moins une fois au cours des } 12 \text { derniers mois }\end{array}$ & $66,0 \%$ & ESCC (2012) \\
\hline \multirow[t]{2}{*}{$\begin{array}{l}\text { Dépistage des } \\
\text { maladies }\end{array}$} & $\begin{array}{l}\text { \% de femmes âgées de } 50 \text { à } 74 \text { ans déclarant avoir subi au moins une mammographie au } \\
\text { cours des cinq dernières années }\end{array}$ & $83,5 \%$ & ESCC (2012) \\
\hline & $\begin{array}{l}\text { \% de femmes âgées de } 25 \text { à } 69 \text { ans déclarant avoir subi au moins un test PAP au cours } \\
\text { des trois dernières années }\end{array}$ & $79,7 \%$ & ESCC (2012) \\
\hline Vaccination (grippe) & $\begin{array}{l}\% \text { de la population âgée de } 12 \text { ans et plus souffrant d'un problème de santé chronique } \\
\text { ayant déclaré avoir reçu un vaccin antigrippal saisonnier au cours des } 12 \text { derniers mois }\end{array}$ & $47,4 \%$ & ESCC (2011-2012) \\
\hline
\end{tabular}




\section{CADRE D'INDICATEURS DES MALADIES CHRONIQUES, STATISTIQUES RAPIDES, ÉDITION PRINTEMPS 2014}

\section{ÉTAT DE SANTÉ GLOBAL ET IMPACTS SUR LA SANTÉ}

Santé générale

\% de la population âgée de 12 ans et plus déclarant avoir une « très bonne » ou une « excellente » santé

$59,9 \%$

ESCC (2011-2012)

\% de la population âgée de 12 ans et plus déclarant avoir une «très bonne » ou une « excellente » santé mentale

$72,2 \%$

ESCC (2011-2012)

Espérance de vie à la naissance

81,7 ans

SCSMC (2006-2008)

Espérance de vie à 65 ans

20,5 ans

SCSMC (2006-2008)

Espérance de vie ajustée en fonction de l'état de santé à la naissance

71,8 ans

SCSMC (2006-2008)

Espérance de vie ajustée en fonction de l'état de santé à 65 ans

15,9 ans

SCSMC (2006-2008)

Morbidité - prévalence \% de la population âgée de 20 ans et plus souffrant d'au moins une maladie chronique importante (cancer, diabète, maladie cardiovasculaire, maladie pulmonaire obstructive chronique)

Prévalence du diabète chez les enfants et les jeunes âgés de 19 ans ou moins

$15,7 \%$

ESCC (2011-2012)

Prévalence du diabète au sein de la population âgée de 20 ans et plus

$0,3 \%$

SCSMC (2008-2009)

Prévalence des maladies cardiaques au sein de la population âgée de 20 ans et plus

$8,7 \%$

SCSMC (2008-2009) $)^{\text {b }}$

Prévalence d'accidents vasculaires cérébraux au sein de la population âgée de 20 ans et plus

$5,5 \%$

ESCC (2011-2012)

Prévalence de l'asthme chez les enfants et les jeunes âgés de 19 ans ou moins

$1,3 \%$

ESCC (2011-2012)

Prévalence de l'asthme au sein de la population âgée de 20 ans et plus

$15,2 \%$

SCSMC (2008-2009)

Prévalence des maladies pulmonaires obstructives chroniques au sein de la population

$8,3 \%$

SCSMC (2008-2009) $)^{\text {b }}$ âgée de 35 ans et plus

Prévalence de l'arthrite au sein de la population âgée de 20 ans et plus

$8,7 \%$

SCSMC (2008-2009)

Prévalence de l'utilisation des services de santé en raison de troubles mentaux chez les enfants et jeunes âgés de 19 ans ou moins

Prévalence de l'utilisation des services de santé en raison de troubles mentaux chez les adultes âgés de 20 ans et plus

Prévalence de troubles anxieux et de l'humeur chez les enfants et jeunes âgés de 19 ans ou moins

Prévalence de troubles anxieux et de l'humeur chez les adultes âgés de 20 ans et plus

Nombre de personnes au sein de l'ensemble de la population vivant avec un cancer ou ayant survécu à un cancer diagnostiqué au cours des 5 dernières années

Nombre de personnes au sein de l'ensemble de la population vivant avec un cancer de la prostate ou ayant survécu à un tel cancer diagnostiqué au cours des 5 dernières années

Nombre de personnes au sein de l'ensemble de la population vivant avec un cancer du poumon ou ayant survécu à un tel cancer diagnostiqué au cours des 5 dernières années

Nombre de personnes au sein de l'ensemble de la population vivant avec un cancer du sein ou ayant survécu à un tel cancer diagnostiqué au cours des 5 dernières années

Nombre de personnes au sein de l'ensemble de la population vivant avec un cancer colorectal ou ayant survécu à un tel cancer diagnostiqué au cours des 5 dernières années

Morbidité - incidence Taux d'incidence du diabète chez les enfants et les jeunes âgés de 19 ans ou moins

Taux d'incidence du diabète au sein de la population âgée de 20 ans et plus

Taux d'incidence de l'asthme chez les enfants et les jeunes âgés de 19 ans ou moins

Taux d'incidence de l'asthme au sein de la population âgée de 20 ans et plus

Taux d'incidence des maladies pulmonaires obstructives chroniques au sein de la population âgée de 35 ans et plus

Taux d'incidence de tous les cancers au sein de la population masculine

Taux d'incidence de tous les cancers au sein de la population féminine

Taux d'incidence du cancer de la prostate au sein de la population masculine

Taux d'incidence du cancer du poumon au sein de la population masculine

Taux d'incidence du cancer du poumon au sein de la population féminine

$17,6 \%$

ESCC (2011-2012)

$8,0 \%$

SCSMC (2008-2009)

$16,2 \%$

SCSMC (2008-2009)

$7,2 \%$

ESCC (2011-2012)

$11,2 \%$

ESCC (2011-2012)

518705 personnes

RCC (2004-2008)

105179 personnes RCC (2004-2008)

29780 personnes

RCC (2004-2008)

90677 personnes

RCC (2004-2008)

67173 personnes

RCC (2004-2008)

42,7 par 100000

SCSMC (2008-2009)

813,6 par 100000 SCSMC (2008-2009)

1 097,8 par 100000 SCSMC (2008-2009)

392,1 par 100000 SCSMC (2008-2009)

930,3 par 100000 SCSMC (2009-2010)

467,5 par $100000^{\text {d }}$ RCC (2007)

364,8 par $100000^{d} \quad$ RCC (2007)

125,8 par $100000^{\text {d }}$ RCC (2007)

69,0 par $100000^{\mathrm{d}}$ RCC (2007)

47,9 par $100000^{d} \quad$ RCC (2007)

60,8 par $100000^{\mathrm{d}} \quad$ RCC (2007)

Taux d'incidence du cancer colorectal au sein de la population masculine

40,9 par $100000^{\text {d }}$ RCC (2007)

Taux d'incidence du cancer colorectal au sein de la population féminine

98,8 par $100000^{\text {d }}$ RCC (2007)

Taux d'incidence du cancer du sein au sein de la population féminine

Suite page suivante 
Annexe A (Suite)

CADRE D'INDICATEURS DES MALADIES CHRONIQUES, STATISTIQUES RAPIDES, ÉDITION PRINTEMPS 2014

\begin{tabular}{|c|c|c|c|}
\hline Multimorbidité & $\begin{array}{l}\text { \% de la population âgée de } 20 \text { ans et plus souffrant de plusieurs maladies chroniques } \\
\text { (au moins } 2 \text { de } 10 \text { maladies chroniques) }\end{array}$ & $14,5 \%$ & ESCC (2011-2012) \\
\hline Incapacité & $\begin{array}{l}\text { \% de la population âgée de } 12 \text { ans et plus déclarant être limitée " parfois » ou " souvent » } \\
\text { dans ses activités en raison de problèmes de santé ou d'une maladie }\end{array}$ & $33,9 \%$ & ESCC (2012) \\
\hline \multirow{3}{*}{ Mortalité } & Taux de mortalité au sein de l'ensemble de la population attribuable aux maladies cardiovasculaires & 203,7 par 100000 & SEC (2009) \\
\hline & Taux de mortalité au sein de l'ensemble de la population attribuable au cancer & 210,9 par 100000 & SEC (2009) \\
\hline & $\begin{array}{l}\text { Taux de mortalité au sein de l'ensemble de la population attribuable aux maladies } \\
\text { respiratoires chroniques }\end{array}$ & 43,5 par 100000 & SEC (2009) \\
\hline \multirow[t]{8}{*}{ Mortalité prématurée } & Années potentielles de vie perdues en raison du cancer & 1504 par 100000 & SEC (2009) \\
\hline & Années potentielles de vie perdues en raison des maladies cardiovasculaires & 755,4 par 100000 & SEC (2009) \\
\hline & Années potentielles de vie perdues en raison des maladies respiratoires chroniques & 118,1 par 100000 & SEC (2009) \\
\hline & Années potentielles de vie perdues en raison du suicide & 362,1 par 100000 & SEC (2009) \\
\hline & $\begin{array}{l}\text { Probabilité de mourir (\%) entre } 30 \text { et } 69 \text { ans de maladies chroniques importantes } \\
\text { (maladies cardiovasculaires, cancer, maladies respiratoires chroniques, diabète) }\end{array}$ & $11,4 \%$ & SEC (2009) \\
\hline & Probabilité de mourir (\%) entre 30 et 69 ans d'une maladie cardiovasculaire & $3,5 \%$ & SEC (2009) \\
\hline & Probabilité de mourir (\%) entre 30 et 69 ans d'un cancer & $7,1 \%$ & SEC (2009) \\
\hline & Probabilité de mourir (\%) entre 30 et 69 ans d'une maladie respiratoire chronique & $0,7 \%$ & SEC (2009) \\
\hline
\end{tabular}

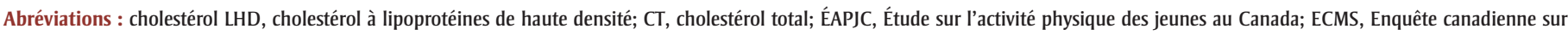

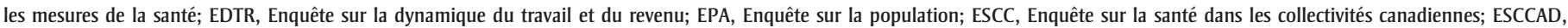

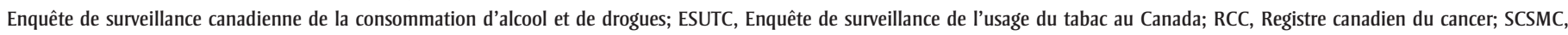
Système canadien de surveillance des maladies chroniques; SEC, statistiques sur l'état civil.

a Tous les taux présentés sont bruts sauf s'il y a indication contraire.

b Des données de l'ESCC de 2011-2012 existent pour cet indicateur et peuvent être ventilées selon des variables démographiques et sociales.

c Multimorbidité : Les maladies chroniques incluses sont les maladies du cœur, les accidents vasculaires cérébraux, les cancers, l'asthme, la maladie pulmonaire obstructive chronique, le diabète, l'arthrite, la maladie d'Alzheimer et autres démences, les troubles anxieux et de l'humeur (dépression).

d Les taux ont été normalisés en fonction de l'âge de la population au Canada en 1991. 


\section{Annexe B : Description et définition des indicateurs}

\section{Table des matières}

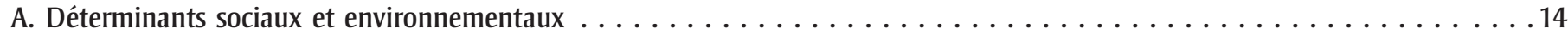

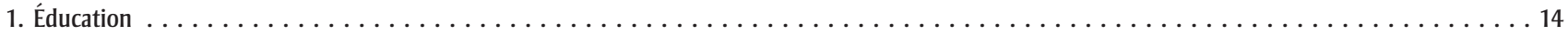

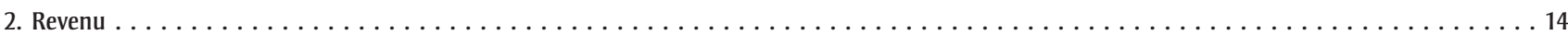

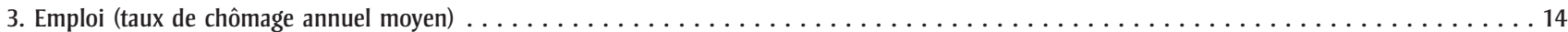

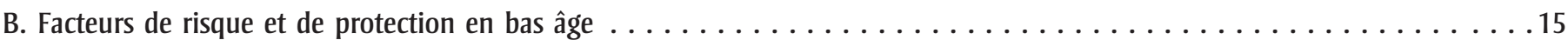

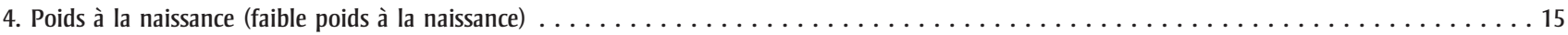

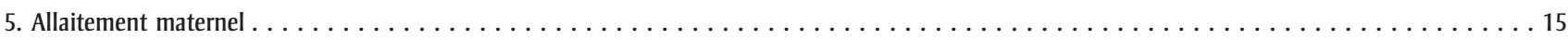

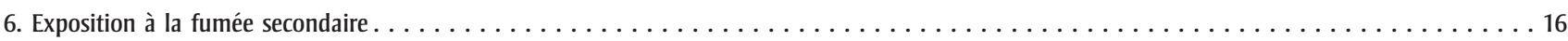

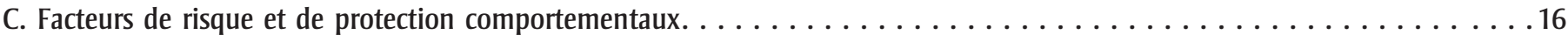

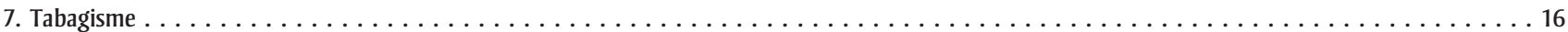

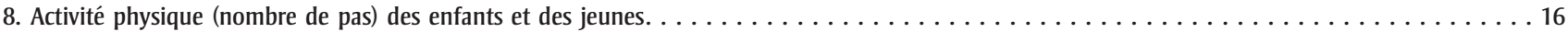

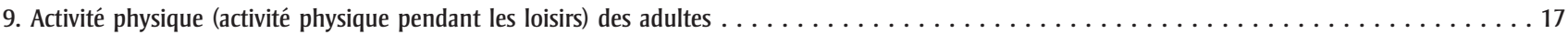

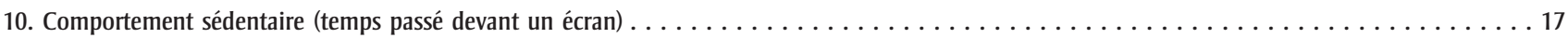

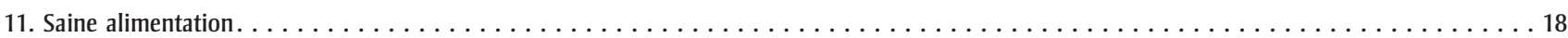

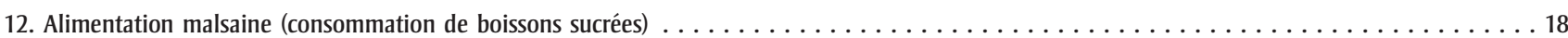

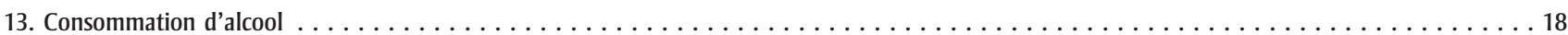

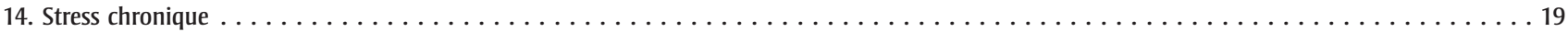

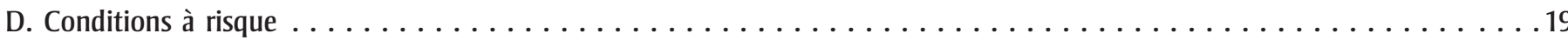

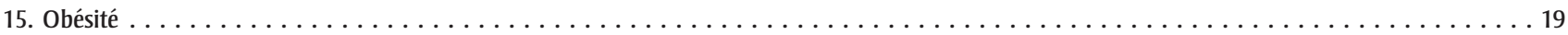

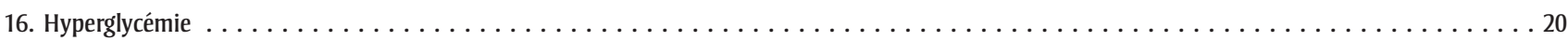

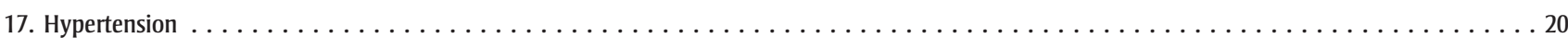

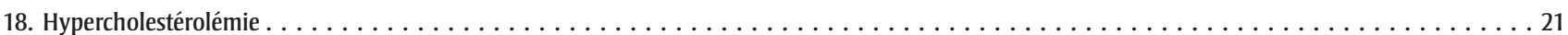

E. Pratiques de prévention des maladies (prévention secondaire) $\ldots \ldots \ldots \ldots$

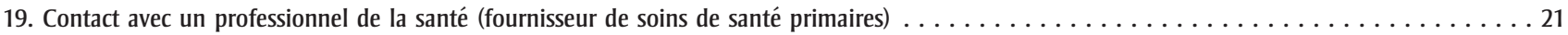

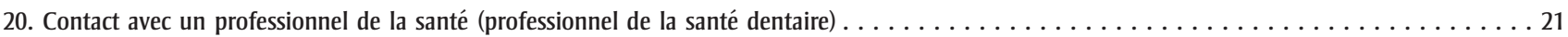

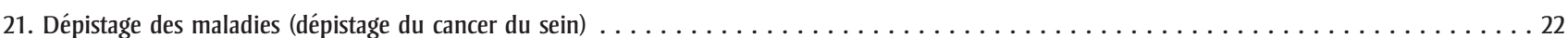

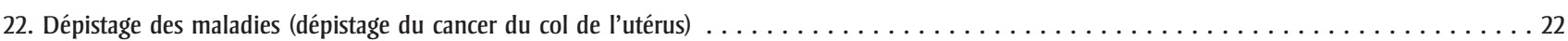

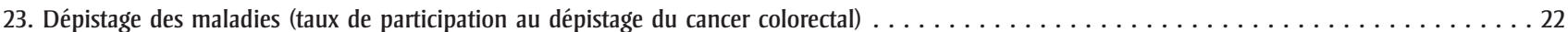

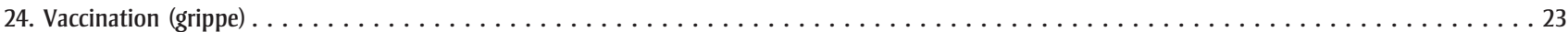

F. État de santé global et impacts sur la santé. . . . . . . . . . . . . . . . . . . . . . . 23

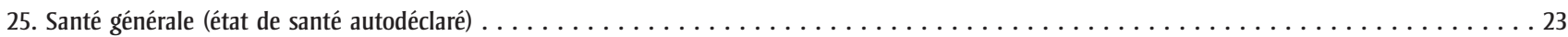

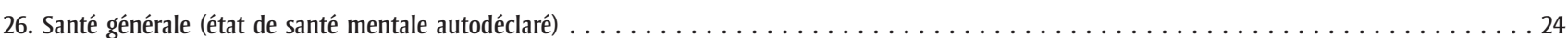

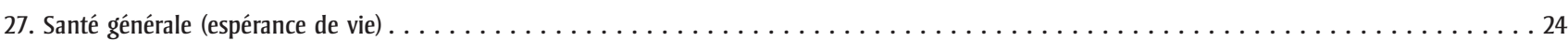

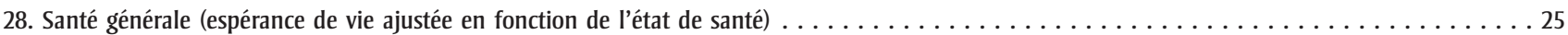

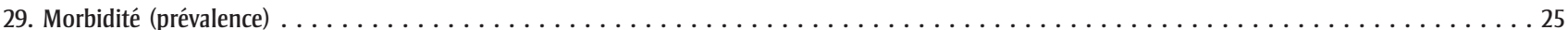

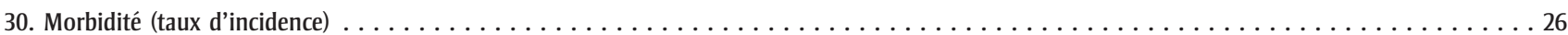

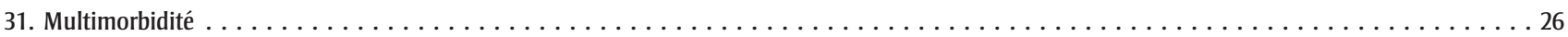

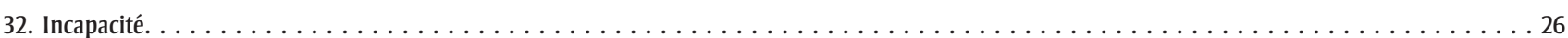

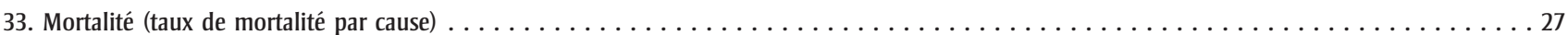

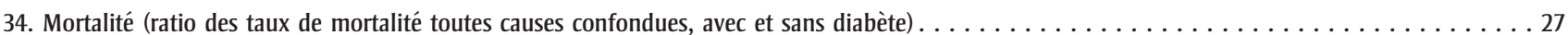

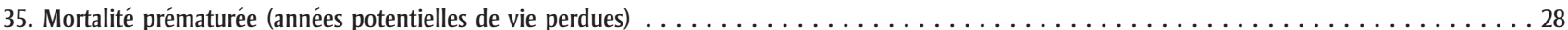

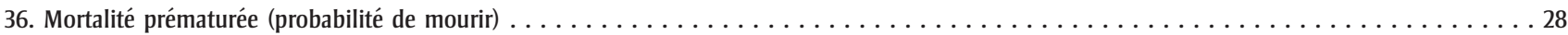




\section{A. Déterminants sociaux et environnementaux}

\section{1. Éducation}

Justification

Mesure

Définition

Source des données

Population

Méthodes de calcul

Remarques supplémentaires

\section{Revenu}

Justification

Définition

Source des données

Population

Méthodes de calcul

Remarques supplémentaires
L'éducation est étroitement liée à la santé de la population et est un déterminant important de la santé ${ }^{1}$

L'éducation contribue à la santé et à la prospérité en :

- fournissant aux personnes les connaissances et les aptitudes nécessaires à la résolution de problèmes,

- contribuant à conférer le sentiment d'avoir une influence et un contrôle sur sa vie,

- augómentant les opportunités d'emploi, la sécurité de revenu et la satisfaction professionnelle,

- améliorant la capacité à accéder à l'information permettant de rester en santé et à la comprendre.

Pourcentage de la population âgée de 20 ans et plus n'ayant pas terminé ses études secondaires.

Pourcentage d'adultes déclarant qu'ils n'ont pas terminé leurs études secondaires, exprimé en tant que proportion de la population totale.

Enquête sur la santé dans les collectivités canadiennes (ESCC), Statistique Canada.

Population âgée de 20 ans et plus.

Numérateur : Nombre de personnes, âgées de 20 ans et plus déclarant qu'elles n'ont pas terminé leurs études secondaires. Dénominateur : Population totale âgée de 20 ans et plus.

On peut interpréter une proportion faible pour cet indicateur comme un résultat positif.

Le revenu est reconnu comme un facteur déterminant de la santé s.2. $^{1,2}$

Le revenu influe sur les conditions de vie, notamment le fait de pouvoir se loger de façon sécuritaire et d'acheter des aliments nutritifs en quantité suffisante. Un faible revenu influe sur le comportement lié à la santé, comme la qualité du régime alimentaire, le niveau d'activité physique et d'autres facteurs de risque. À long terme, un faible revenu a une incidence négative sur la santé, car il diminue la capacité de faire des choix sains et de vivre pleinement au quotidien. Un faible revenu prive la personne de l'accès à un logement décent, à l'éducation, au transport et à d'autres facteurs nécessaires à une pleine participation à la vie. Les contraintes de la pauvreté peuvent être particulièrement dommageables ${ }^{2}$. Un faible revenu est associé à l'augmentation de la prévalence du risque de maladies chroniques et à une prévalence de conditions chroniques plus élevée que dans la population totale. Les données démontrent clairement un lien entre la pauvreté et une mauvaise santé ainsi que la présence de maladies chroniques.

Pourcentage de la population vivant sous les seuils de faible revenu (SFR) après impôt.

Proportion de la population dont le revenu est inférieur au SFR et qui consacre une part disproportionnée des revenus du ménage après impôt aux dépenses de première nécessité (alimentation, logement et vêtements) par rapport à la famille canadienne moyenne.

Enquête sur la dynamique du travail et du revenu (EDTR), Statistique Canada.

Population totale.

Numérateur : Nombre de personnes vivant dans un ménage dont le revenu après impôt est inférieur au SFR. Dénominateur : Population totale.

Le recours au SFR est une méthode sûre et largement reconnue d'estimation de la population vivant dans des conditions de pauvreté ou proches de la pauvreté au Canada ${ }^{3}$. Cette approche permet d'estimer le nombre de familles (et par le fait même, de personnes) qui consacrent une part disproportionnée du revenu de leur ménage après impôt aux dépenses de première nécessité (alimentation, logement et vêtements) par rapport à la famille canadienne moyenne.

On peut interpréter une proportion faible pour cet indicateur comme un résultat positif.

\section{Emploi (taux de chômage annuel moyen)}

Justification

Le chômage, le sous-emploi ou encore un travail stressant ou dangereux sont associés à une piètre santé2.

L'emploi a un effet marqué sur la santé physique, mentale et sociale. Le travail rémunéré permet non seulement de gagner de l'argent, mais il confère aussi un sentiment d'identité et d'utilité, permet d'avoir des contacts sociaux et des possibilités de croissance personnelle ${ }^{1}$. Perdre ces avantages peut avoir une incidence négative sur sa santé et sur celle de sa famille. Le chômage mène souvent à des difficultés matérielles et à la pauvreté, puisqu'il diminue le revenu et les autres avantages sociaux. La perte d'emploi constitue un événement stressant pouvant avoir une influence sur l'estime de soi et pouvant contribuer à l'augomentation des soucis et de l'anxiété, lesquels, à leur tour, risquent d'augomenter la possibilité qu'une personne réagisse aux difficultés en adoptant des comportements néfastes pour la santé comme l'usage de la cigarette ou la consommation excessive d'alcool.

De façon générale, les personnes sans emploi ont une espérance de vie moins élevée et sont plus souvent atteintes de troubles de santé que les personnes ayant un emploi.

Mesure

Taux de chômage annuel moyen (part de la population active âgée de 15 ans et plus ne travaillant pas au cours de la période de référence) .

Définition Proportion de la population active âgée de 15 ans et plus sans emploi (mais en recherche d'emploi) au cours de l'année précédente.

Source des données

Enquête sur la population active (EPA), Statistique Canada.

Population

Population âgée de 15 ans et plus. 


\section{Emploi (taux de chômage annuel moyen) [Suite]}

\begin{tabular}{ll}
\hline Méthodes de calcul & $\begin{array}{l}\text { Numérateur : Nombre de personnes faisant partie de la population active, sans emploi (mais en recherche d'emploi) au } \\
\text { cours de l'année précédente. } \\
\text { Dénominateur : Population active (voir les remarques ci-dessous). }\end{array}$ \\
Remarques supplémentaires & $\begin{array}{l}\text { La population active est formée de la population âgée de } 15 \text { ans et plus ayant un emploi ou étant au chômage. Elle n'inclut } \\
\text { ni les personnes qui ne travaillent pas et ne prévoient pas de retourner au travail, ni celles qui ne sont pas disponibles pour } \\
\text { un emploi et n'en cherchent pas. } \\
\text { On peut interpréter une proportion faible pour cet indicateur comme un résultat positif. }\end{array}$ \\
\hline
\end{tabular}

\section{B. Facteurs de risque et de protection en bas âge}

\begin{tabular}{|c|c|}
\hline Justification & $\begin{array}{l}\text { Le poids à la naissance est un indicateur de la santé générale des nouveau-nés et un déterminant clé de la survie des } \\
\text { nourrissons, de leur santé et de leur développement. } \\
\text { Les bébés ayant un faible poids à la naissance risquent davantage de mourir en bas âge, d'avoir des maladies d'enfance graves } \\
\text { et des séquelles à long terme (p. ex. l'invalidité). Un faible poids à la naissance est associé à une faible croissance durant } \\
\text { l'enfance et un risque plus élevé de développer le diabète de type } 2 \text { et des maladies cardiovasculaires plus tard dans la vie }{ }^{4-6} \text {. }\end{array}$ \\
\hline Mesure & Pourcentage de naissances vivantes associées à un faible poids à la naissance. \\
\hline Définition & Pourcentage des naissances vivantes associées à un poids à la naissance inférieur à 2500 g sur l'ensemble des naissances vivantes. \\
\hline Population & Ensemble des naissances vivantes. \\
\hline Méthodes de calcul & $\begin{array}{l}\text { Numérateur : Nombre des naissances vivantes associées à un poids à la naissance inférieur à } 2500 \mathrm{~g} \text {. } \\
\text { Dénominateur : Nombre des naissances vivantes associées à un poids à la naissance connu. }\end{array}$ \\
\hline Remarques supplémentaires & $\begin{array}{l}\text { Un faible poids à la naissance est défini par l'Organisation mondiale de la santé comme un poids inférieur à } 2500 \text { g à la naissance. } \\
\text { Le faible poids à la naissance peut être attribuable à une naissance prématurée, un retard de croissance intra-utérin ou les deux? } \\
\text { On peut interpréter une proportion faible pour cet indicateur comme un résultat positif. }\end{array}$ \\
\hline Mesure & $\begin{array}{l}\text { Pourcentage de femmes âgées de } 15 \text { ans et plus déclarant avoir nourri leur enfant exclusivement au sein pendant les } 6 \\
\text { premiers mois ou plus. }\end{array}$ \\
\hline Définition & $\begin{array}{l}\text { Pourcentage de femmes ayant accouché dans les cinq dernières années et déclarant avoir eu recours exclusivement à } \\
\text { l'allaitement maternel pour leur dernier enfant pendant six mois et plus. }\end{array}$ \\
\hline
\end{tabular}

Suite page suivante 


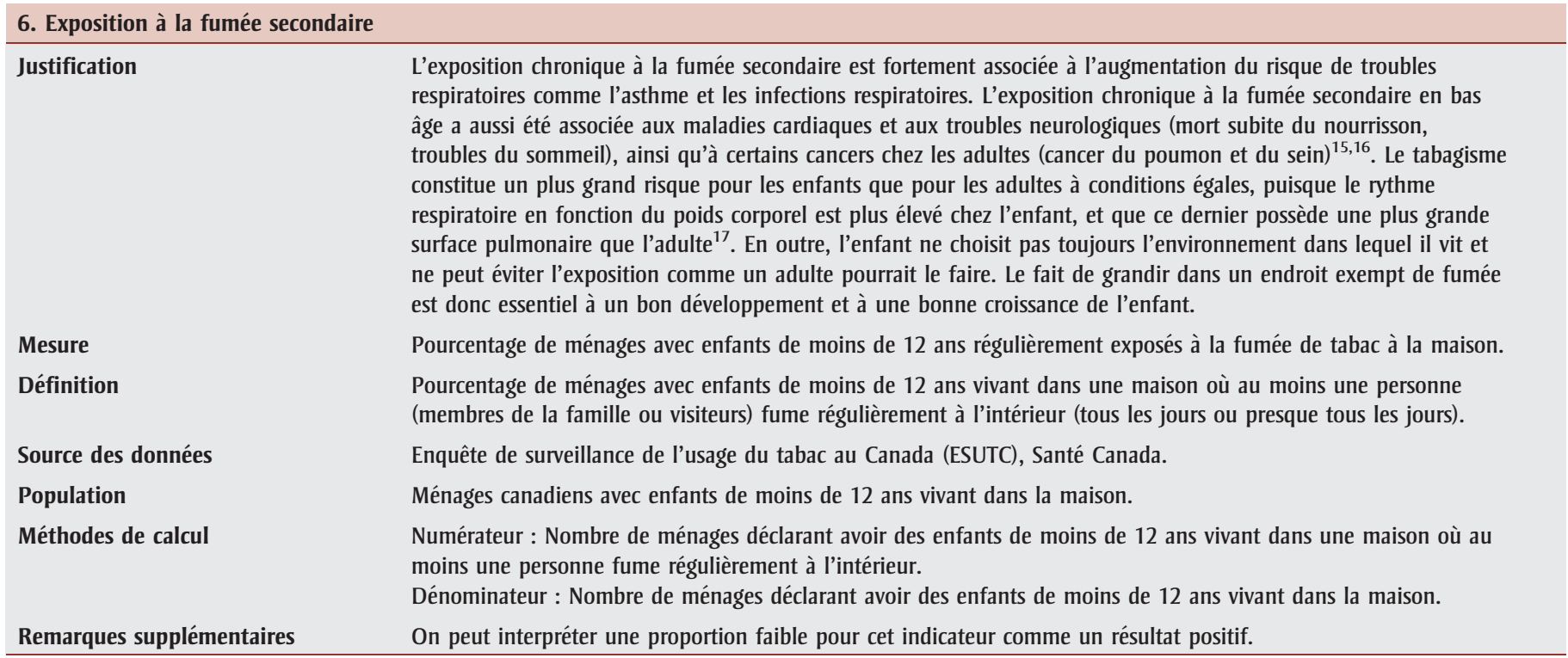

\section{Facteurs de risque et de protection comportementaux}

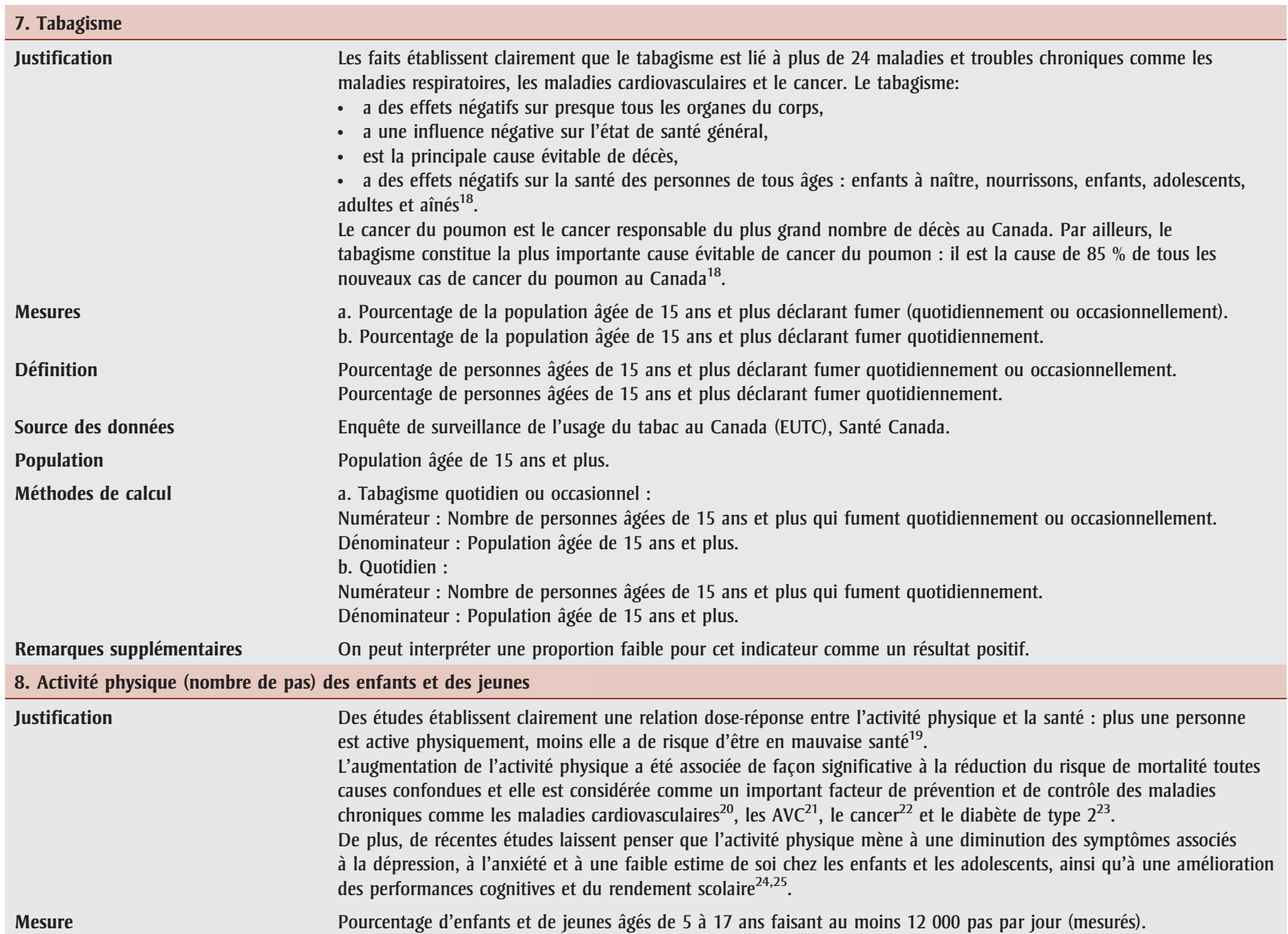

Suite page suivante 


\section{Activité physique (nombre de pas) des enfants et des jeunes [Suite]}

\section{Définition}

Source des données

Population

Méthodes de calcul

Remarques supplémentaires

Cet indicateur mesure le pourcentage d'enfants et de jeunes âgés de 5 à 17 ans faisant au moins 12000 pas par jour chaque jour de la semaine.

Étude sur l'activité physique des jeunes au Canada (EAPJC), Institut canadien de la recherche sur la condition physique et le mode de vie.

Enfants et jeunes âgés de 5 à 17 ans.

Cet indicateur représente le pourcentage d'enfants et de jeunes ayant fait au moins 12000 pas par jour chaque jour de la semaine. Les pas ont été comptés à l'aide d'un podomètre pendant sept jours ${ }^{26,27}$.

Les Directives canadiennes en matière d'activité physique recommandent que, pour obtenir un bienfait pour la santé, les enfants et les jeunes de 5 à 17 ans pratiquent au moins 60 minutes d'activité physique d'intensité modérée à intense par jour ${ }^{28}$. Les podomètres mesurent divers types d'activité physique réalisés dans tous les contextes, notamment les loisirs, le déplacement vers l'école, les sports, les tâches ménagères et le travail. Il a été démontré que la mesure de 12000 pas par jour peut servir d'indicateur substitutif permettant d'évaluer la conformité aux directives canadiennes ${ }^{29}$. On reconnaît qu'il s'agit d'une estimation prudente, car certains types d'activité physique, comme la nage et l'exercice à bicyclette, se mesurent mal à l'aide de podomètres. Les podomètres sont des instruments qui ne sont pas aussi sensibles au pas que les accéléromètres.

On peut interpréter une proportion élevée pour cet indicateur comme un résultat positif.

\section{Activité physique (activité physique pendant les loisirs) des adultes}

Justification

Des études établissent clairement une relation dose-réponse entre l'activité physique et la santé : plus une personne est active physiquement, moins elle a de risque d'être en mauvaise santé ${ }^{19}$.

L'augmentation de l'activité physique a été associée de façon significative à la réduction du risque de mortalité toutes causes confondues et elle est considérée comme un important facteur de prévention et de contrôle des maladies chroniques comme les maladies cardiovasculaires ${ }^{20}$, les $\mathrm{AVC}^{21}$, le cancer ${ }^{22}$ et le diabète de type $2^{23-25}$.

Mesure

Pourcentage de la population âgée de 20 ans et plus déclarant être « active » ou « modérément active » physiquement pendant ses loisirs.

Définition

Pourcentage de personnes âgées de 20 ans et plus classées comme « actives » ou « modérément actives » selon l'Indice de l'activité physique dans les loisirs (IAPL).

Source des données

Enquête sur la santé dans les collectivités canadiennes (ESCC), Statistique Canada.

Population

Population âgée de 20 ans et plus.

Méthodes de calcul

Numérateur : Nombre de personnes âgées de 20 ans et plus physiquement « actives » ou « modérément actives » pendant les loisirs.

Dénominateur : Population totale âgée de 20 ans et plus.

Remarques supplémentaires

L'IAPL est une mesure mixte qui classe les personnes comme « actives », « modérément actives » ou « inactives » en fonction de leurs réponses à des questions posées sur la quantité totale d'énergie par jour dépensée au cours d'activités de loisirs au cours des trois derniers mois. Il s'agit d'un indicateur substitutif de l'ensemble de l'activité physique. On peut interpréter une proportion élevée pour cet indicateur comme un résultat positif.

\section{Comportement sédentaire (temps passé devant un écran)}

Justification

Mesure

Définition

Source des données

Population

Méthodes de calcul
II semble que le comportement sédentaire ait des effets physiologiques directs sur le métabolisme et la santé vasculaire $^{30,31}$. Une relation dose-réponse a été établie entre la durée du comportement sédentaire et l'augmentation du taux de mortalité toutes causes confondues, ainsi que du taux de mortalité attribuable aux maladies cardiovasculaires ${ }^{32}$. Le fait de regarder la télévision et d'utiliser un ordinateur sont les comportements sédentaires les plus étudiés, et de nombreuses enquêtes permettent d'obtenir des données à propos de ces activités. Selon des études récentes, le temps passé devant un écran (télévision, ordinateur ou jeux vidéo) est associé à l'inactivité durant les loisirs, à une mauvaise alimentation et à l'obésité ${ }^{33}$. Un temps excessif passé devant un écran augogmente le risque d'obésité et de prise de poids, peu importe le niveau d'activité physique, de même que le risque d'accidents cardiovasculaires et de décès ${ }^{34,35}$.

Pourcentage de la population âgée de 12 ans et plus déclarant passer plus de 14 heures par semaine à regarder la télévision ou à utiliser l'ordinateur pendant leurs loisirs.

Proportion de la population âgée de 12 ans et plus déclarant passer plus de 14 heures par semaine à regarder la télévision ou à utiliser leur ordinateur pendant leurs loisirs.

Enquête sur la santé dans les collectivités canadiennes (ESCC), Statistique Canada.

Population âgée de 12 ans et plus.

Numérateur : Nombre de personnes âgées de 12 ans et plus déclarant passer plus de 14 heures par semaine à regarder la télévision, des vidéos ou à utiliser un ordinateur pendant leur temps libre. Le temps d'ordinateur comprend les jeux vidéo et l'utilisation d'Internet.

Dénominateur : Population totale âgée de 12 ans et plus. 


\section{Comportement sédentaire (temps passé devant un écran) [Suite]}

\section{Remarques supplémentaires Cette mesure ne comprend pas les activités sédentaires telles que la lecture ou le sommeil.}

On a établi des Directives canadiennes sur le comportement sédentaire pour les enfants et les jeunes âgés de 0 à 17 ans. Ces lignes directrices recommandent que les enfants et les jeunes âgés de 5 à 17 ans passent au maximum deux heures par jour devant un écran pendant leurs loisirs ${ }^{36}$. Cet indicateur fournit une estimation prudente du nombre d'enfants qui ne respectent pas ces directives. Le temps de sédentarité associé aux adultes n'a pas encore été clairement défini, mais on a établi dans la littérature à deux heures par jour le seuil au-delà duquel le risque de maladie chronique $\mathrm{s}^{\text {'accroitt }}{ }^{37}$. On peut interpréter une proportion faible pour cet indicateur comme un résultat positif.

11. Saine alimentation

Justification

Une saine alimentation peut aider à prévenir et à contrôler les maladies et les troubles chroniques comme l'hypertension, l'obésité li,39 $^{38}$, les maladies cardiovasculaires ${ }^{40}$, le diabète l1 $^{41}$ et l'ostéoporose ${ }^{42,43}$. Une saine alimentation a aussi été associée à une diminution de la mortalité toutes causes confondues ${ }^{44}$. La consommation quotidienne de fruits et légumes est considérée comme un indicateur de la qualité de l'alimentation ${ }^{45}$.

Mesure

Définition

Source des données

Population

Méthodes de calcul

Remarques supplémentaires
Pourcentage de la population âgée de 12 ans et plus déclarant consommer des fruits et légumes au moins cinq fois par jour.

Pourcentage de personnes déclarant consommer des fruits et légumes généralement au moins cinq fois par jour.

Enquête sur la santé dans les collectivités canadiennes (ESCC), Statistique Canada.

Population âgée de 12 ans et plus.

Numérateur : Nombre de personnes âgées de 12 ans et plus déclarant consommer des fruits et légumes au moins cinq fois par jour.

Dénominateur : Population totale âgée de 12 ans et plus.

Des études de validation indiquent que cet indicateur substitutif est fiable et peut servir à quantifier l'apport en fruits et légumes (c. à d. le nombre de portions par jour) et à fournir une approximation de la qualité de l'alimentation ${ }^{46}$. Cet indicateur n'est PAS une mesure en conformité avec le Guide alimentaire canadien ${ }^{47}$.

On peut interpréter une proportion élevée pour cet indicateur comme un résultat positif.

\section{Alimentation malsaine (consommation de boissons sucrées)}

Justification

Mesure

Définition

Source des données

Population

Méthodes de calcul

Remarques supplémentaires
Dans les dernières décennies, la consommation de boissons sucrées a considérablement augrmenté partout dans le monde. La consommation de ces boissons, particulièrement les boissons gazeuses et les boissons aux fruits car elles ont une faible valeur nutritive et ont peu d'effet sur le sentiment de satiété, ont été associées à un apport énergétique trop élevé. De vastes études de cohorte et des études expérimentales démontrent une forte association positive entre une grande consommation de boissons sucrées et une prise de poids ou une obésité, et ce, autant chez les enfants que chez les adultes ${ }^{48}$.

Pourcentage de la population âgée de 5 à 19 ans déclarant consommer des boissons sucrées tous les jours.

Pourcentage d'enfants et de jeunes déclarant consommer des boissons sucrées tous les jours.

Enquête canadienne sur les mesures de la santé (ECMS), Statistique Canada.

Population âgée de 5 à 19 ans.

Numérateur : Nombre de personnes âgées de 5 à 19 ans déclarant consommer des boissons sucrées au moins une fois par jour, tous les jours.

Dénominateur : Population totale âgée de 5 à 19 ans.

Les enfants faisaient partie de la catégorie des buveurs quotidiens de boissons sucrées (c. à d. boissons gazeuses ordinaires, boissons sportives ou boissons fruitées) si leur consommation quotidienne moyenne était égale ou supérieure à une boisson sucrée par jour. Le Guide alimentaire canadien ${ }^{47}$ recommande de restreindre les boissons très calorifiques telles que les boissons aromatisées aux fruits, les boissons gazeuses, les boissons sportives et énergisantes ainsi que les boissons sucrées chaudes ou froides.

On peut interpréter une proportion faible pour cet indicateur comme un résultat positif.

\section{Consommation d'alcool}

Justification

D'après l'Oróanisation mondiale de la santé, la consommation excessive d'alcool est le troisième plus important facteur de risque de maladies chroniques dans les pays développés ${ }^{49}$. La consommation excessive d'alcool durant une longue période est associée à l'augmentation du risque de maladies chroniques comme les maladies du foie, certains cancers, les maladies cardiovasculaires (cardiopathie hypertensive, cardiopathie ischémique, AVC) et le décès prématuré ${ }^{50}$. Les directives canadiennes de consommation d'alcool à faible risque ont été élaborées afin d'aider les Canadiens à modérer leur consommation d'alcool et à réduire les conséquences immédiates et à long terme liées à la consommation d'alcool ${ }^{51}$.

Mesure

Pourcentage de la population âgée de 15 ans et plus déclarant boire davantage d'alcool que ce que préconisent les directives de consommation d'alcool à faible risque à long terme.

Définition

Pourcentage de personnes âgées de 15 ans et plus déclarant boire davantage d'alcool que ce que préconisent les directives de consommation à faible risque pour la santé à long terme.

Source des données

Enquête de surveillance canadienne de la consommation d'alcool et de drogues (ESCCAD), Santé Canada.

Population Population âgée de 15 ans et plus. 

les directives canadiennes de consommation à faible risque à long terme.

Dénominateur : Population totale âgée de 15 ans et plus.

Remarques supplémentaires

Les dernières directives canadiennes sur la consommation d'alcool à faible risque visant à réduire les risques à long terme pour la santé recommandent un maximum de 10 verres* standards par semaine pour les femmes et un maximum de 15 verres standards par semaine pour les hommes ${ }^{51}$.

* Un verre standard équivaut à 13,6 g d'alcool.

On peut interpréter une proportion faible pour cet indicateur comme un résultat positif.

\section{Stress chronique}

Justification

L'exposition au stress chronique, un état de tension prolongé causé par des facteurs stressants internes ou externes, déclenche dans l'organisme des changements biochimiques et physiologiques prévisibles qui nuisent aux systèmes nerveux et immunitaire ${ }^{52}$.

Des données épidémiologiques permettent de démontrer que le stress chronique est associé à l'apparition de nombreuses maladies chroniques fréquentes ${ }^{53,54}$. II a été prouvé que le stress chronique fait augómenter le rythme cardiaque et la tension artérielle, ce qui finit par avoir des conséquences graves, notamment des maladies cardiovasculaires (infarctus du myocarde, insuffisance cardiaque, AVC) ${ }^{55,56}$ et des maladies mentales. En outre, l'augmentation de l'exposition au stress peut mener à une faible capacité d'adaptation et à des comportements nocifs pour la santé, par exemple le tabagisme, la consommation excessive d'alcool et les mauvaises habitudes alimentaires, qui nuisent à l'état de santé et contribuent à l'apparition de maladies chroniques.

Mesure

Pourcentage de la population âgée de 12 ans et plus déclarant que la plupart de ses journées ont été « assez stressantes » ou " extrêmement stressantes » au cours des 12 derniers mois.

Définition

Pourcentage de personnes âgées de 12 ans et plus déclarant que la plupart de leurs journées ont été « assez stressantes » ou « extrêmement stressantes » au cours des 12 derniers mois (plutôt que " pas du tout stressantes », " pas très stressantes » ou « un peu stressantes »).

Source des données

Population

Enquête sur la santé dans les collectivités canadiennes (ESCC), Statistique Canada.

Méthodes de calcul Population âgée de 12 ans et plus.

Numérateur : Nombre de personnes âgées de 12 ans et plus déclarant que la plupart de leurs journées ont été « assez stressantes » ou " extrêmement stressantes » au cours des 12 derniers mois. Dénominateur : Population totale âgée de 12 ans et plus.

Remarques supplémentaires

La perception d'une vie stressante est utilisée comme indicateur substitutif du stress chronique et cette mesure englobe les personnes ayant perçu la plupart de leurs journées comme assez «stressantes » ou " extrêmement stressantes » au cours de l'année précédente.

On peut interpréter une proportion faible pour cet indicateur comme un résultat positif.

\section{Conditions à risque}

\section{Obésité}

Justification

L'obésité est définie comme « une accumulation excessive de graisse corporelle pouvant poser un risque pour la santé $»^{57}$. L'obésité constitue un facteur de risque pour de nombreux troubles chroniques comme les maladies cardiovasculaires, certains types de cancers, le diabète de type 2, l'arthrose, les troubles de santé mentale et d'autres facteurs négatifs sur la santé ${ }^{58-60}$. Chez les enfants, il a été démontré que l'excès de graisse corporelle est en corrélation avec des effets négatifs sur la santé, autant à court terme qu'à long terme ${ }^{61,62}$.

L'obésité constitue l'un des principaux problèmes de santé au Canada. L'Organisation mondiale de la santé considère que l'obésité se classe au cinquième rang des facteurs de risque de décès toutes causes confondues.

La principale cause de l'obésité et de l'embonpoint est le déséquilibre entre l'énergie consommée et celle dépensée.

Mesures

a. Pourcentage d'enfants et de jeunes âgés de 5 à 17 ans qui sont obèses (donnée mesurée).

b. Pourcentage de la population âgée de 18 à 79 ans qui est obèse (donnée mesurée).

Définition

Pourcentage de la population classée comme obèse. Chez les adultes, l'obésité est définie comme un indice de masse corporelle (IMC) supérieur ou égal à $30,0 \mathrm{~kg} / \mathrm{m}^{2}$. Chez les enfants et les jeunes, l'obésité est définie en fonction des seuils de l'OMS de 2007 établis selon l'âge et le sexe ${ }^{63}$.

Source des données

Enquête canadienne sur les mesures de la santé (ECMS), Statistique Canada.

Population

Enfants et jeunes : Population âgée de 5 à 17 ans.

Adultes : Population âgée de 18 ans et plus.

Méthodes de calcul

Numérateur : Nombre de personnes classées comme obèses d'après leur IMC. Dénominateur : Population totale. 

c'est le seuil fixe d'IMC de $30 \mathrm{~kg} / \mathrm{m}^{2}$ qui est utilisé pour définir l'obésité. II n'existe pas de valeur fixe chez les enfants, étant donné la variabilité de l'IMC au cours de la croissance, mais des seuils d'IMC fonction de l'âge et du sexe qui ont été calculés dans des populations de référence.

On peut interpréter une proportion faible pour cet indicateur comme un résultat positif.

\begin{tabular}{|c|c|}
\hline \multicolumn{2}{|l|}{ 16. Hyperǵlycémie } \\
\hline Justification & $\begin{array}{l}\text { Une hyperglycémie persistante peut conduire à des lésions des petits vaisseaux sanguins (p. ex. une néphropathie, } \\
\text { une neuropathie ou une rétinopathie diabétique) et à des complications des grands vaisseaux sauguins (p. ex. } \\
\text { une coronaropathie, une maladie vasculaire périphérique ou un } A V C)^{64} \text {. Le diabète est un trouble chronique caractérisé } \\
\text { par l'incapacité de l'organisme à produire ou à utiliser l'insuline, ce qui cause une hyperglycémie. Même à des } \\
\text { niveaux inférieurs au seuil de diagnostic du diabète, l'hyperglycémie persistante peut mener à l'apparition de } \\
\text { troubles comme la coronaropathie ou l'AVC }{ }^{65,66} \text {. } \\
\text { Le dépistage précoce de l'hyperglycémie chez les patients non diagnostiqués et le contrôle rigoureux de la glycémie } \\
\text { chez les patients ayant un diagnostic clinique de diabète contribue à la diminution de la progression des lésions des } \\
\text { petits vaisseaux sanguins }{ }^{67} \text { et peut réduire les difficultés associées au diabète et à ses complications }{ }^{68-70} \text {. }\end{array}$ \\
\hline Mesure & Pourcentage de la population âgée de 20 à 79 ans ayant une glycémie élevée (mesurée). \\
\hline Définition & $\begin{array}{l}\text { Pourcentage de personnes âgées de } 20 \text { à } 79 \text { ans présentant une glycémie élevée, soit une concentration sérique de } \\
\text { glucose à jeun supérieure à } 7,0 \mathrm{mmol} / \mathrm{L} \text {. }\end{array}$ \\
\hline Source des données & Enquête canadienne sur les mesures de la santé (ECMS), Statistique Canada. \\
\hline Population & Population âgée de 20 à 79 ans. \\
\hline \multicolumn{2}{|l|}{ 17. Hypertension } \\
\hline Justification & $\begin{array}{l}\text { L'hypertension artérielle peut mener à des lésions vasculaires et représente un facteur de risque important de } \\
\text { maladies cardiovasculaires précoces (AVC, coronaropathie, insuffisance cardiaque et maladie vasculaire périphérique) } \\
\text { De nombreuses études montrent que les décès attribuables aux maladies cardiovasculaires augmentent progressivement } \\
\text { avec l'augogmentation de la tension artérielle, et ce, à partir de niveaux aussi bas que } 115 / 75 \mathrm{~mm} \mathrm{Hg} \mathrm{Hg}^{73} \text {. La prévention } \\
\text { et le contrôle de l'hypertension, par l'intermédiaire de changements dans les habitudes de vie ou par la médication, } \\
\text { peuvent conduire à une diminution signnificative du risque d'AVC ou de coronaropathie } \mathrm{e}^{74,75} \text {. }\end{array}$ \\
\hline Population & Population âgée de 20 à 79 ans. \\
\hline Méthodes de calcul & $\begin{array}{l}\text { Numérateur : Nombre de personnes âgées de } 20 \text { à } 79 \text { ans présentant de l'hypertension. } \\
\text { Dénominateur : Population totale âgée de } 20 \text { à } 79 \text { ans. }\end{array}$ \\
\hline Remarques supplémentaires & $\begin{array}{l}\text { La définition de l'hypertension (c. à d. tension systolique supérieure ou égale à } 140 \mathrm{~mm} \text { Hg et tension diastolique } \\
\text { supérieure ou égale à } 90 \mathrm{~mm} \mathrm{Hg} \text { ) se fonde sur les recommandations de } 2012 \text { du Programme éducatif canadien } \\
\text { sur l'hypertension }{ }^{76} \text {, lesquelles correspondent aux lignes directrices du septième rapport du Comité national mixte } \\
\text { (CNM7) sur la classification et le traitement de la tension artérielle chez l'adulte }{ }^{77} \text {. } \\
\text { Cet indicateur tient compte des personnes chez qui on a mesuré une tension artérielle élevée au cours d'une seule } \\
\text { visite médicale, indépendamment d'un diagnostic d'hypertension. Cette mesure exclut l'hypertension gravidique } \\
\text { (liée à la grossesse). Cet indicateur ne tient pas compte des personnes dont l'hypertension a été diagnostiquée et qui } \\
\text { est bien stabilisée. II ne peut donc servir d'indicateur de prévalence de l'hypertension. } \\
\text { Une ventilation supplémentaire est établie en fonction de l'existence d'une hypertension diagnostiquée } \\
\text { (autodéclaration qu'un diagnostic d'hypertension a été préalablement posé par un professionnel de la santé ou } \\
\text { utilisation de médicaments en raison d'une hypertension). } \\
\text { On peut interpréter une proportion faible pour cet indicateur comme un résultat positif. }\end{array}$ \\
\hline
\end{tabular}




\section{Hypercholestérolémie}

Justification

Mesure

Définition

Source des données

Population

Méthodes de calcul

Remarques supplémentaires
Le rapport entre le cholestérol total (CT) et le cholestérol à lipoprotéines de haute densité (LHD) permet de prévoir le risque de coronaropathie ainsi que d'indiquer un métabolisme anormal du cholestérol ${ }^{78}$. Le rapport entre le CT et le LHD est une manière simple, non effractive et économique de prédire l'existence et l'ampleur de l'athérosclérose coronaire; il est également un indicateur de risque de troubles cardiovasculaires (coronaropathie, accident ischémique cérébral ${ }^{79,80}$ ) et de résistance à l'insuline ${ }^{81}$. Le risque d'accident cardiaque est considérablement plus élevé lorsque le ratio CT/C-LHD est égal ou supérieur à $5 \mathrm{mmol} / \mathrm{L}^{82,83}$.

Pourcentage de la population âgée de 20 à 79 ans ayant un taux de cholestérol élevé (ratio cholestérol total sur cholestérol LHD [CT/C-LHD]) (donnée mesurée).

Pourcentage de personnes âgées de 20 à 79 ans participant à une enquête transversale fondée sur un échantillon représentatif à l'échelle nationale et présentant une concentration élevée de cholestérol sanguin déterminée à l'aide d'un ratio de CT/ C-LHD supérieur ou égal à $5 \mathrm{mmol} / \mathrm{L}$.

Enquête canadienne sur les mesures de la santé (ECMS), Statistique Canada.

Population âgée de 20 à 79 ans.

Numérateur : Nombre de personnes âgées de 20 à 79 ans présentant un ratio de CT/C-LHD élevé à jeun. Dénominateur : Nombre de personnes âgées de 20 à 79 ans ayant subi un dosage à jeun du cholestérol total et du cholestérol LHD pour en déterminer le ratio.

Le seuil de 5,0 mmol/L du ratio CT/C-LHD a été choisi en fonction des recommandations des lignes directrices canadiennes en matière de diagnostic et de traitement de la dyslipidémie ainsi que de prévention des maladies cardiovasculaires chez l'adulte ${ }^{83,84}$.

Cet indicateur tient compte des personnes chez lesquelles on a mesuré un ratio CT/C-LHD élevé dans un échantillon sanguin unique prélevé à jeun indépendamment d'un diagnostic d'hypercholestérolémie. II ne tient pas compte des personnes dont l'hypercholestérolémie a été diagnostiquée et qui est bien stabilisée. Il ne peut donc servir d'indicateur de l'hypercholestérolémie diagnostiquée.

Une ventilation supplémentaire en fonction de l'existence d'une hypercholestérolémie fournit le taux d'hypercholestérolémie (ratio CT/C-LHD élevé) diagnostiquée (autodéclaration d'hypercholestérolémie préalablement décelée par un professionnel de la santé).

On peut interpréter une proportion faible pour cet indicateur comme un résultat positif.

\section{E. Pratiques de prévention des maladies (prévention secondaire)}

\section{Contact avec un professionnel de la santé (fournisseur de soins de santé primaires)}

\begin{tabular}{|c|c|}
\hline Justification & $\begin{array}{l}\text { Le fait d'établir une relation continue avec un fournisseur de soins primaires joue un rôle important dans le maintien } \\
\text { d'une bonne santé et garantit les meilleurs soins de santé, notamment le dépistage préventif, le traitement précoce } \\
\text { et un meilleur contrôle des maladies chroniques }{ }^{85} \text {. L'accès aux soins primaires favorise aussi la promotion de la santé, } \\
\text { par exemple des conseils relatifs à un mode de vie sain et des consultations liées à la santé mentale }{ }^{86} \text {. Un accès } \\
\text { régulier à un médecin de famille peut mener à l'amélioration de l'état de santé et à la réduction des frais de soins de } \\
\text { santé, en raison de la diminution de la consultation de spécialistes et de l'utilisation des services hospitaliers. }\end{array}$ \\
\hline Mesure & $\begin{array}{l}\text { Pourcentage de la population âgée de } 12 \text { ans et plus déclarant avoir consulté un médecin de famille ou un } \\
\text { omnipraticien au moins une fois au cours des } 12 \text { derniers mois. }\end{array}$ \\
\hline Définition & $\begin{array}{l}\text { Pourcentage de personnes déclarant avoir consulté un fournisseur de soins primaires (c. à d. un médecin de famille } \\
\text { ou un omnipraticien) au moins une fois au cours des } 12 \text { derniers mois pour obtenir des soins ou des conseils sur leur } \\
\text { santé physique, émotionnelle ou mentale. }\end{array}$ \\
\hline Source des données & Enquête sur la santé dans les collectivités canadiennes (ESCC), Statistique Canada. \\
\hline Population & Population âgée de 12 ans et plus. \\
\hline Méthodes de calcul & $\begin{array}{l}\text { Numérateur : Nombre de personnes âgées de } 12 \text { ans et plus déclarant avoir consulté un fournisseur de soins } \\
\text { de santé primaires au moins une fois au cours des } 12 \text { derniers mois. } \\
\text { Dénominateur : Population totale âgée de } 12 \text { ans et plus. }\end{array}$ \\
\hline Remarques supplémentaires & On peut interpréter une proportion élevée pour cet indicateur comme un résultat positif. \\
\hline \multicolumn{2}{|c|}{ 20. Contact avec un professionnel de la santé (professionnel de la santé dentaire) } \\
\hline Justification & $\begin{array}{l}\text { La santé bucco-dentaire est un facteur important d'un bon état de santé général. Des visites régulières chez le } \\
\text { dentiste sont essentielles au diagnostic précoce et à la prévention de troubles bucco-dentaires comme la } \\
\text { carie et la parodontopathie. L'Association dentaire canadienne a constaté que les Canadiens n'ayant pas régulièrement } \\
\text { accès à des soins dentaires ont une moins bonne santé bucco-dentaire et un état de santé général inférieur à ceux qui } \\
\text { ont un accès régulier à ces services }{ }^{87} \text {. }\end{array}$ \\
\hline
\end{tabular}




\section{Contact avec un professionnel de la santé (professionnel de la santé dentaire) [Suite]}

\begin{tabular}{ll}
\hline Mesure & $\begin{array}{l}\text { Pourcentage de la population âgée de } 12 \text { ans et plus déclarant avoir consulté un dentiste, un hygiéniste dentaire } \\
\text { ou un orthodontiste au moins une fois au cours des } 12 \text { derniers mois. } \\
\text { Dourcentage de personnes déclarant avoir consulté un professionnel de la santé dentaire (dentiste, hygiéniste } \\
\text { dentaire ou orthodontiste) au moins une fois au cours des } 12 \text { derniers mois. } \\
\text { Source des données }\end{array}$ \\
$\begin{array}{l}\text { Enquête sur la santé dans les collectivités canadiennes (ESCC), Statistique Canada. } \\
\text { Population âgée de } 12 \text { ans et plus. }\end{array}$ \\
Méthodes de calcul & $\begin{array}{l}\text { Numérateur : Nombre de personnes âgées de } 12 \text { ans et plus déclarant avoir consulté un professionnel de la santé } \\
\text { dentaire au moins une fois au cours des } 12 \text { derniers mois. } \\
\text { Dénominateur : Population totale âgée de } 12 \text { ans et plus. } \\
\text { On peut interpréter une proportion élevée pour cet indicateur comme un résultat positif. }\end{array}$
\end{tabular}

21. Dépistage des maladies (dépistage du cancer du sein)

Justification Le cancer de sein constitue la forme la plus répandue de cancer chez les femmes canadiennes et il est également la deuxième cause de décès attribuable à un cancer ${ }^{88}$. Le dépistage du cancer du sein est généralement perçu comme une intervention en santé profitable, en particulier chez les femmes âgées de 50 à 74 ans. Les données provenant de vastes études expérimentales et d'études démographiques démontrent clairement que le dépistage par mammographie réduit les décès causés par le cancer du sein de 25 à $30 \%{ }^{89,90}$.

Mesure

Pourcentage de femmes âgées de 50 à 74 ans déclarant avoir subi au moins une mammographie au cours des cinq dernières années.

Définition

Pourcentage de la population cible (femmes de 50 à 74 ans) déclarant avoir subi une mammographie au cours des cinq dernières années.

Source des données

Enquête sur la santé dans les collectivités canadiennes (ESCC), Statistique Canada.

Population

Population ciblée par le dépistage : Femmes âgées de 50 à 74 ans.

Méthodes de calcul

Numérateur : Nombre de femmes âgées de 50 à 74 ans ayant eu au moins une mammographie au cours des cinq dernières années.

Dénominateur : Nombre total de femmes âgées de 50 à 74 ans.

Remarques supplémentaires

L'intervalle de cinq ans de cet indicateur NE concorde PAS avec les lignes directrices nationales en vigueur, soit " un dépistage régulier à l'aide d'une mammographie tous les 2 à 3 ans » destiné aux femmes de 25 à 69 ans présentant un risque moyen ${ }^{91,92}$. Les données actuelles ne fournissent qu'une estimation de la population déclarant avoir eu une mammographie au cours des cinq dernières années.

On peut interpréter une proportion élevée pour cet indicateur comme un résultat positif.

\section{Dépistage des maladies (dépistage du cancer du col de l'utérus)}

Justification

Définition

Source des données

Population

Méthodes de calcul

Remarques supplémentaires

Le fait de subir un test de Papanicolaou (PAP) de façon régulière est associé à la diminution de l'incidence du cancer du col de l'utérus et du risque de décès lié à ce type de cancer ${ }^{93}$. Le test PAP permet de détecter les lésions précancéreuses avant qu'elles ne deviennent cancéreuses ou encore de déceler la maladie à un stade précoce, au moment où le traitement est le plus efficace.

Même si le cancer invasif du col de l'utérus peut facilement être prévenu, il demeure le $13^{\mathrm{e}}$ cancer en importance chez les femmes canadiennes tous âges confondus. Le dépistage inadéquat ou l'absence de dépistage ont été reconnus comme les principaux facteurs responsables de cette situation ${ }^{94}$.

Pourcentage de femmes âgées de 25 à 69 ans déclarant avoir subi au moins un test PAP au cours des trois dernières années.

Pourcentage de femmes âgées de 25 à 69 ans ayant subi au moins un test PAP au cours des trois dernières années, comme les lignes directrices canadiennes les plus récentes le recommandent.

Enquête sur la santé dans les collectivités canadiennes (ESCC), Statistique Canada.

Femmes âgées de 25 à 69 ans.

Numérateur : Nombre de femmes âgées de 25 à 69 ans ayant subi au moins un test PAP au cours des trois dernières années. Dénominateur : Nombre total de femmes âgées de 25 à 69 ans (à l'exception des femmes ayant subi une hystérectomie).

Les recommandations des lignes directrices canadiennes en matière de dépistage du cancer du col de l'utérus visent les femmes asymptomatiques sexuellement actives ou ayant été sexuellement actives ${ }^{95}$. Les lignes directrices canadiennes de 2013 pour le dépistage du col de l'utérus recommandent aux femmes âgées de 25 à 69 ans un dépistage de routine aux trois ans.

Cet indicateur peut être utilisé pour estimer le taux associé à un recours au dépistage du cancer du col de l'utérus et inclut les femmes non sexuellement actives ou ne l'ayant jamais été.

On peut interpréter une proportion élevée pour cet indicateur comme un résultat positif.

\section{Dépistage des maladies (taux de participation au dépistage du cancer colorectal)}

\section{Justification}

Le cancer colorectal constitue le deuxième cancer en importance chez les hommes canadiens et le troisième chez les femmes, et il est également la deuxième cause de décès attribuable à un cancer ${ }^{96}$. Le dépistage peut réduire l'incidence du cancer colorectal (détection au stade précancéreux) et le risque de décès (détection à un stade précoce) ${ }^{97}$.

Suite page suivante 


\section{Dépistage des maladies (taux de participation au dépistage du cancer colorectal) [Suite]}

Mesure

Source des données

Population

Méthodes de calcul

Remarques supplémentaires
Définition

Pourcentage de la population âgée de 50 à 74 ans ayant subi au moins un test de recherche de sang occulte dans les selles (TRSOS), une colonoscopie ou une siǵmoïdoscopie au cours de la période recommandée.

Pourcentage de personnes âgées de 50 à 74 ans déclarant avoir subi au moins un TRSOS au cours des deux dernières années ou une colonoscopie ou une siǵmoïdoscopie au cours des 5 dernières années.

Enquête sur la santé dans les collectivités canadiennes (ESCC), Statistique Canada.

Population âgée de 50 à 74 ans.

Numérateur : Nombre de personnes âgées de 50 à 74 ans ayant subi un dépistage du cancer colorectal au moins une fois au cours de la période recommandée (moins de 2 ans dans le cas de la RSOS et moins de 5 ans dans le cas de la colonoscopie ou de la sigmoïdoscopie).

Dénominateur : Population totale âgée de 50 à 74 ans.

Cet indicateur peut être utilisé pour estimer le taux d'utilisation du dépistagé du cancer colorectal. Selon les lignes directrices canadiennes les plus récentes en matière de dépistage du cancer colorectal, des données probantes étayent le recours à un test de recherche de sang occulte dans les selles tous les ans ou tous les deux ans, et des preuves acceptables appuient le recours à une sigmoïdoscopie dans le cadre de l'examen médical périodique chez les personnes asymptomatiques âgées de plus de 50 ans.

Chez les personnes associées à un risque normal, la colonoscopie n'est généralement pas utilisée comme premier test de dépistage du cancer colorectal.

On peut interpréter une proportion élevée pour cet indicateur comme un résultat positif.

\section{Vaccination (grippe)}

\section{Justification}

Le taux de complications, le nombre d'admissions à l'hôpital et les décès causés par une maladie transmissible comme la grippe sont plus élevés chez les adultes atteints de maladies chroniques graves ${ }^{98}$. La vaccination annuelle contre la grippe peut :

- prévenir l'apparition de la grippe,

- aider à contrôler un grave épisode de grippe s'il survient,

- généralement faciliter le contrôle et la gestion des complications liées à une maladie chronique ${ }^{99}$

Afin de réduire la morbidité et la mortalité associées à la grippe, le Comité consultatif national de l'immunisation (CCNI) recommande que les programmes de vaccination ciblent notamment les personnes très à risque de complications liées à la grippe. Selon les recommandations du CCNI pour l'année 2011-2012, les maladies chroniques pouvant le plus mener à des complications liées à la grippe sont les troubles cardiaques et pulmonaires, le diabète et les autres maladies métaboliques ainsi que le cancer et les troubles affaiblissant le système immunitaire (en raison de l'affection sous-jacente et/ou d'un traitement, etc.) ${ }^{100}$.

Mesure

Pourcentage de la population âgée de 12 ans et plus souffrant d'un problème de santé chronique* ayant déclaré avoir reçu un vaccin antigrippal saisonnier au cours des 12 derniers mois.

Définition

Source des données

Population

Méthodes de calcul

Proportion de personnes âgées de 12 ans et plus souffrant d'une maladie chronique et déclarant avoir reçu une immunisation contre la grippe (vaccin antigrippal) au cours des 12 derniers mois.

Enquête sur la santé dans les collectivités canadiennes (ESCC), Statistique Canada.

Population âgée de 12 ans et plus souffrant d'une maladie chronique.

Numérateur : Nombre de personnes âgées de 12 ans et plus souffrant d'une maladie chronique* et déclarant avoir reçu un vaccin contre la grippe au cours des 12 derniers mois.

Dénominateur : Population totale âgée de 12 ans et plus souffrant d'une maladie chronique*.

Remarques supplémentaires

respiratoires chroniques (asthme et MPOC), diabète et cancer (tous les types).

On peut interpréter une proportion élevée pour cet indicateur comme un résultat positif.

\section{F. État de santé gólobal et impacts sur la santé}

\section{Santé générale (état de santé autodéclaré)}

Justification

Mesure

Définition
L'état de santé autodéclaré permet de mesurer la perception qu'a une personne de son état de santé global. Les études montrent que l'évaluation qu'une personne fait de son état de santé générale constitue un bon indicateur de morbidité et de mortalité, même après contrôle de divers indicateurs sociodémographiques, psychosociaux et physiques relatifs à l'état de santé ${ }^{101}$. Une mauvaise perception de son état de santé est associée à la présence de maladies chroniques, d'un certain degré d'invalidité, de douleurs et de comportements nocifs pour la santé comme un faible degré d'activité physique ou le tabagisme ${ }^{102,103}$.

Pourcentage de la population âgée de 12 ans et plus déclarant avoir une " très bonne » ou une « excellente » santé.

Pourcentage de personnes déclarant avoir une «très bonne » ou une « excellente » santé en proportion de la population totale âgée de 12 ans et plus. 


\section{Santé générale (état de santé autodéclaré) [Suite]}

\begin{tabular}{ll}
\hline Source des données & Enquête sur la santé dans les collectivités canadiennes (ESCC), Statistique Canada. \\
Population & Population âgée de 12 ans et plus. \\
Méthodes de calcul & Numérateur : Nombre de personnes âgées de 12 ans et plus déclarant avoir une « très bonne » ou une « excellente » santé. \\
& Dénominateur : Population totale âgée de 12 ans et plus.
\end{tabular}

Remarques supplémentaires L'état de santé autodéclaré est une mesure substitutive de l'état de santé gilobal.

On peut interpréter une proportion élevée pour cet indicateur comme un résultat positif.

\section{Santé générale (état de santé mentale autodéclaré)}

Justification

Mesure

Définition

Source des données

Population

Méthodes de calcul

Remarques supplémentaires
La santé mentale et la santé physique ont toutes deux une incidence sur l'état de santé général. Aujourd'hui, le mieux-être en matière de santé mentale ne se définit plus seulement par l'absence de maladie mentale, tels les troubles mentaux, les problèmes émotionnels ou la détresse, mais aussi par la présence de facteurs comme les dispositions à aimer la vie, l'atteinte d'un équilibre dans les divers aspects de sa vie et la capacité d'adaptation ${ }^{104}$. Il existe des influences bidirectionnelles entre les troubles de santé mentale et les maladies chroniques. Les troubles de santé mentale, plus particulièrement la dépression et l'anxiété, précèdent souvent l'apparition de maladies chroniques. En outre, les personnes atteintes depuis longtemps d'une maladie chronique ont plus de risque de développer des problèmes de santé mentale et font état de degrés plus élevés de détresse ${ }^{105,106}$.

Pourcentagé de la population âgée de 12 ans et plus déclarant avoir une « très bonne » ou une « excellente » santé mentale.

Pourcentage de personnes ayant autoévalué leur santé mentale comme « excellente » ou « très bonne » en proportion de la population totale âgée de 12 ans et plus.

Enquête sur la santé dans les collectivités canadiennes (ESCC), Statistique Canada.

Population âgée de 12 ans et plus.

Numérateur : Nombre de personnes âgées de 12 ans et plus déclarant leur santé mentale comme « très bonne » ou " excellente ».

Dénominateur : Population totale âgée de 12 ans et plus.

Les études laissent penser que l'état de santé mentale autodéclaré est fortement lié à la détresse psychologique, aux symptômes dépressifs, à la limitation des activités et au fonctionnement social physique et émotionnel ${ }^{107}$. Cet indicateur est donc considéré comme une mesure substitutive de la santé mentale générale d'une personne et constitue un indicateur de l'état de santé global.

On peut interpréter une proportion élevée pour cet indicateur comme un résultat positif.

\section{Santé générale (espérance de vie)}

Justification

L'espérance de vie est largement utilisée au Canada et ailleurs dans le monde afin de mesurer l'état de santé général d'une population ${ }^{108}$. L'espérance de vie permet de prédire le nombre d'années qu'il reste à vivre ${ }^{109}$. Deux mesures sont généralement utilisées : l'espérance de vie à la naissance, qui décrit la santé d'une population envisagée comme un tout, et l'espérance de vie à 65 ans qui reflète l'état de santé des aînés.

Mesures

a. Espérance de vie à la naissance.

b. Espérance de vie à 65 ans.

Définition

L'espérance de vie correspond au nombre moyen d'années qu'une personne pourrait s'attendre à vivre, d'après un ensemble de taux de mortalité selon l'âge au cours d'une période d'observation donnée.

L'espérance de vie à 65 ans correspond au nombre moyen d'années que ceux qui atteignent 65 ans pourraient s'attendre à vivre, d'après un ensemble de taux de mortalité selon l'âge au cours d'une période donnée.

Source des données

Population

Système canadien de surveillance des maladies chroniques (SCSMC), Agence de la santé publique du Canada.

Population totale.

L'espérance de vie à la naissance et celle à 65 ans sont calculées à l'aide de tables de survie fournissant une analyse transversale de la mortalité et de la survie d'une population pour une période donnée (trois ans). Les tables d'espérance de vie sont calculées en fonction des probabilités de mortalité. La méthode utilisée pour calculer cet indicateur est la méthode de Chiang ${ }^{110}$. La table de survie fait appel à 19 groupes d'âge standards (moins de 1 an, 1 à 4 ans, 5 à 9 ans, ..., 80 à 84 ans, 85 ans et plus). La fonction de Gompertz sert à obtenir une estimation précise de l'espérance de vie du dernier intervalle, qui est ouvert, à savoir celui des 85 ans et plus $^{111}$. Les estimations de mortalité se fondent sur des données de mortalité sur trois ans.

Remarques supplémentaires
L'espérance de vie à la naissance est le nombre moyen d'années qu'un nouveau-né peut s'attendre à vivre si les taux de mortalité selon l'âge ayant cours pour une année précise s'appliquent.

L'espérance de vie à 65 ans représente le nombre moyen d'années que les personnes atteignant l'âge de 65 ans peuvent s'attendre à vivre.

On peut interpréter une proportion élevée (en années) pour cet indicateur comme un résultat positif. 
28. Santé générale (espérance de vie ajustée en fonction de l'état de santé)

Justification

L'espérance de vie ajustée en fonction de l'état de santé (EVAS) représente le nombre d'années potentielles de vie à vivre en pleine santé, pour un âge déterminé. Cette mesure combine les données relatives à la morbidité et à la mortalité en un seul indicateur de la santé d'une population. Elle n'est donc pas seulement une mesure de la durée de vie, mais aussi une mesure de la qualité de vie ${ }^{112}$. L'EVAS à la naissance est souvent comparée à l'espérance de vie afin de déterminer le nombre d'années vécues en mauvaise santé ou avec une mauvaise qualité de vie ${ }^{112}$.

Mesures

a. Espérance de vie ajustée en fonction de l'état de santé à la naissance.

b. Espérance de vie ajustée en fonction de l'état de santé à 65 ans.

Définition

L'EVAS représente le nombre d'années potentielles de vie équivalant aux années à vivre en pleine santé, compte tenu des profils moyens dans une population ${ }^{113}$.

Source des données

Système canadien de surveillance des maladies chroniques (SCSMC), Agence de la santé publique du Canada. Enquête sur la santé dans les collectivités canadiennes (ESCC), Statistique Canada.

Population

Méthodes de calcul

Population totale.

L'EVAS combine les mesures de l'état de santé selon le sexe et selon l'âge ainsi que la mortalité selon le sexe et selon l'âge (période de trois ans) en une seule statistique. La méthode adaptée de Sullivan ${ }^{114}$, un prolongement de la méthode des tables de survie, est utilisée pour calculer cette mesure. La table de survie fait appel à 19 groupes d'âge standards (moins de 1 an, 1 à 4 ans, 5 à 9 ans, ..., 80 à 84 ans, 85 ans et plus). La fonction de Gompertz sert à obtenir une estimation exacte de l'espérance de vie pour le dernier intervalle, qui est ouvert, à savoir celui des 85 ans et plus ${ }^{111}$. La variable " années vécues », une variable de la nomenclature de la table de survie standard, est corrigée en fonction de la mesure de la qualité de vie associée à l'état de santé, à savoir l'Indice de l'état de santé (HUI3 ou Health Utility Index Mark 3) provenant des données de l'ESCC. La correction permet de faire une distinction entre les années vécues en bonne santé et les années vécues en mauvaise santé. Le nombre d'années vécues en bonne santé permet de calculer l'EVAS. La mortalité est quant à elle estimée en fonction des données de mortalité sur trois ans.

Remarques supplémentaires L'EVAS est une mesure du nombre moyen d'années de vie en bonne santé qu'une personne peut s'attendre à vivre. On peut interpréter une proportion élevée (en années) pour cet indicateur comme un résultat positif.

\section{Morbidité (prévalence)}

Justification

La prévalence permet d'évaluer le nombre total de personnes souffrant d'une maladie particulière dans une population à un moment donné ou pour une période donnée ${ }^{115}$. Elle est directement influencée par le nombre de cas d'apparition de cette maladie (incidence), par la progression de celle-ci et par le taux de survie dans la population. Le taux de prévalence d'une maladie peut continuer d'augmenter dans une population alors que le taux d'incidence demeure stable, si les gens vivent plus longtemps qu'avant avec la maladie en question, notamment en raison de l'amélioration des traitements et du contrôle de la maladie.

Mesure

Pourcentage de la population souffrant d'une ou de plusieurs maladies chroniques.

Définition

Proportion de personnes souffrant d'une ou de plusieurs maladies chroniques au sein d'une population au cours d'une période donnée. Cet indicateur est composé de plusieurs mesures, qui représentent chacune la prévalence d'une maladie chronique* particulière dans la population, à un moment donné ou pour une période donnée.

Source des données Système canadien de surveillance des maladies chroniques (SCSMC), Agence de la santé publique du Canada. Enquête sur la santé dans les collectivités canadiennes (ESCC), Statistique Canada.

Population

L'âge de la population varie en fonction de la maladie :

- Population d'enfants et de jeunes âgés de 19 ans ou moins pour le diabète, l'asthme et les troubles mentaux;

- Population âgée de 20 ans et plus pour le diabète, les maladies cardiaques, l'AVC, l'asthme, les troubles mentaux et l'arthrite;

- Population âgée de 35 ans et plus pour la MPOC.

Méthodes de calcul

Numérateur : Nombre de personnes souffrant d'une maladie chronique particulière* dans la population à un moment donné. Dénominateur : Population totale à un moment donné.

Remarques supplémentaires

*Les maladies chroniques sont les suivantes : diabète, maladies cardiovasculaires (maladies cardiaques, insuffisance cardiaque, cardiopathies ischémiques, et AVC), maladies respiratoires chroniques (asthme, MPOC), arthrite, maladie mentale (tous les troubles mentaux, troubles anxieux et de l'humeur) et cancers (ensemble des cancers, cancer de la prostate, cancer du sein, cancer colorectal et cancer des poumons). En outre, la fréquence des personnes présentant au moins une maladie chronique grave (cancer, diabète, maladies cardiovasculaires, maladies respiratoires chroniques) est mesurée.

Les estimations sont calculées à l'aide de données autodéclarées (ESCC) et de données administratives (SCSMC). La fréquence de l'utilisation d'un service permettant de diagnostiquer les troubles mentaux est utilisée comme mesure substitutive pour la prévalence des maladies mentales.

La prévalence du cancer est mesurée à partir de la prévalence de cancer sur cinq ans à l'échelle individuelle, soit le nombre de personnes vivant avec un cancer ou ayant combattu un cancer diagnostiqué au cours des cinq ans précédant la date de référence.

Tous les taux présentés sont des taux bruts, à l'exception des tendances pour lesquelles les taux sont normalisés en fonction de l'âge pour tenir compte du Recensement du Canada de 1991.

On peut interpréter une proportion faible de cet indicateur comme un résultat positif.

Suite page suivante 


\section{Morbidité (taux d'incidence)}

Justification

Mesure

Définition

Source des données

Population

Méthodes de calcul

Remarques supplémentaires

\section{Multimorbidité}

Justification

Mesures

Définition

Source des données

Population

Méthodes de calcul

Remarques supplémentaires

32. Incapacité

Justification

Mesure

Définition

Source des données

Population
L'incidence mesure le nombre de nouvelles personnes souffrant d'une maladie donnée dans une population durant une période donnée. L'incidence est beaucoup plus sujette à des changements dans le temps (tendances) que la prévalence ${ }^{116}$. Le taux d'incidence est influencé par deux groupes de facteurs principaux :

- le taux sous-jacent d'occurrence d'une maladie, qui reflète la prévalence des facteurs de risque et donc le succès des efforts de prévention primaire,

- le taux de détection et de diagnostic d'une maladie chronique donnée, qui peut être influencé par l'intensité et l'efficacité du dépistage de la maladie ou des programmes de prévention.

Taux d'incidence d'une ou de plusieurs maladies chroniques.

Taux de nouveaux cas diagnostiqués d'une maladie particulière* par 100000 habitants.

Système canadien de surveillance des maladies chroniques (SCSMC), Agence de la santé publique du Canada.

La limite d'âge inférieure de la population varie en fonction de la maladie :

- Population d'enfants et de jeunes âgés de 19 ans ou moins pour le diabète et l'asthme;

- Population âgée de 20 ans et plus pour le diabète et l'asthme;

- Population âgée de 35 ans et plus pour la MPOC;

- Population totale en fonction du sexe pour le cancer.

Numérateur : Nombre de nouveaux cas d'une maladie chronique donnée* diagnostiqués dans la population pour une année donnée.

Dénominateur : Population totale à risque de cette maladie pour une année donnée.

*Les maladies chroniques sont : diabète, insuffisance cardiaque, cardiopathies ischémiques,asthme, MPOC et cancer (tous les cancers, cancer de la prostate, cancer du sein, cancer colorectal et cancer des poumons).

Tous les taux présentés sont des taux bruts, à l'exception des tendances pour lesquelles les taux sont normalisés en fonction de l'âge pour tenir compte du Recensement du Canada de 1991.

On peut interpréter une proportion faible de cet indicateur comme un résultat positif.

La multimorbidité correspond à la présence simultanée d'au moins 2 maladies chroniques sans que l'un des troubles ne prédomine nécessairement.

La multimorbidité est de plus en plus reconnue comme un prédicteur indépendant d'incapacité, de mauvaise qualité de vie, de complications liées à un traitement, de coûts de soins de santé élevés et d'augomentation du risque de mortalitéé ${ }^{117,118}$.

a. Pourcentage de la population âgée de 20 ans et plus souffrant de plusieurs maladies chroniques (au moins 2 de 10 maladies chroniques*).

b. Pourcentage de la population âgée de 20 ans et plus souffrant de plusieurs maladies chroniques (au moins 3 de 10 maladies chroniques*).

Proportion de personnes âgées de 20 ans et plus souffrant de plus d'une maladie chronique ( 2 ou plus, ou 3 ou plus, selon le cas $^{*}$ ) au sein de la population.

Enquête sur la santé dans les collectivités canadiennes (ESCC), Statistique Canada.

Population âgée de 20 ans et plus.

Numérateur : Nombre de personnes âgées de 20 ans et plus souffrant d'au moins 2 (ou d'au moins 3) maladies chroniques sur $10^{*}$.

Dénominateur : Population totale âgée de 20 ans et plus.

*Les maladies chroniques sont les suivantes : maladies cardiaques, AVC, cancer, asthme, MPOC, diabète, arthrite, maladie d'Alzheimer et autres démences, troubles anxieux et de l'humeur (dépression).

On peut interpréter une proportion faible pour cet indicateur comme un résultat positif.

La maladie chronique peut mener à des limitations fonctionnelles pouvant nuire à la capacité d'une personne à accomplir ses activités ou ses tâches quotidiennes à la maison, à l'école ou au travail ${ }^{119,120}$. L'incapacité à court ou à long terme a une incidence sociale négative sur la qualité de vie et la capacité à prendre soin de soi-même et de sa famille ${ }^{121}$. De plus, elle peut avoir des impacts économiques négatifs directs dans le cas d'une perte de revenu pour les périodes non travaillées ${ }^{122}$.

Pourcentage de la population âgée de 12 ans et plus déclarant être limitée «parfois » ou « souvent » dans ses activités en raison de problèmes de santé ou d'une maladie.

Proportion de personnes âgées de 12 ans et plus ayant répondu « parfois » ou « souvent » à la question : " Un problème physique, mental ou de santé à long terme réduit-il la quantité ou le type d'activité que vous pouvez faire à la maison, au travail, à l'école ou durant d'autres activités, par exemple les déplacements et les loisirs? ».

Enquête sur la santé dans les collectivités canadiennes (ESCC), Statistique Canada.

Population âgée de 12 ans et plus. 


\section{Incapacité [Suite]}

\section{Méthodes de calcul}

Remarques supplémentaires
Numérateur : Nombre de personnes âgées de 12 ans et plus déclarant être « parfois » ou « souvent » limitées dans leurs activités en raison d'une maladie*.

Dénominateur : Population totale âgée de 12 ans et plus.

*Les personnes doivent indiquer que leur état physique, leur état mental ou leur problème de santé à long terme est causé par une maladie chronique ou un état émotionnel/mental (c.-à-d. à l'exception des contraintes attribuables au vieillissement, à des accidents, à des troubles congénitaux ou génétiques, etc.).

Dans le cas de cet indicateur, la limitation d'activité est une mesure substitutive permettant de mesurer

l'incapacité attribuable à une maladie.

On peut interpréter une proportion faible pour cet indicateur comme un résultat positif.
33. Mortalité (taux de mortalité par cause)

Justification

Mesure

Définition

Source des données

Population

Méthodes de calcul

Remarques supplémentaires
Le taux de mortalité est largement utilisé comme mesure de la santé d'une population ${ }^{123,124}$. L'étude des tendances, des taux et des causes de mortalité en lien avec certaines maladies chroniques, ainsi que celle de la façon dont les décès varient en fonction des populations peuvent permettre d'évaluer les possibilités à long terme de réduire les décès causés par des maladies chroniques et peuvent offrir un aperçu de la manière dont améliorer :

- les conditions sociales et environnementales,

- les tendances relatives aux facteurs de risque sous-jacents,

- les interventions médicales ${ }^{125}$.

Taux de mortalité au sein de l'ensemble de la population attribuable à une ou plusieurs maladies chroniques*.

Nombre de décès causés au sein d'une population par une ou plusieurs maladies chroniques*, par 100000 personnes pour une année donnée.

Statistique de l'état civil (SEC), Statistique Canada.

Population totale.

Numérateur : Nombre de décès dans la population en raison d'une ou de plusieurs maladies chroniques* sélectionnées. Dénominateur : Population totale.

*Maladies sélectionnées : maladies cardiaques, cancer, maladies respiratoires chroniques, ainsi que suicide. Le calcul est réalisé pour chacun des groupes de maladies séparément et pour l'ensemble des trois grandes catégories de maladies chroniques (c.-à-d. maladies cardiovasculaires, cancer et maladies respiratoires chroniques).

Tous les taux présentés sont des taux bruts, à l'exception des tendances pour lesquelles les taux sont normalisés en fonction de l'âge pour tenir compte du Recensement du Canada de 1991.

On peut interpréter une proportion faible pour cet indicateur comme un résultat positif.

34. Mortalité (ratio des taux de mortalité toutes causes confondues, avec et sans diabète)

Justification

L'analyse des statistiques en matière de mortalité se fondant exclusivement sur la cause immédiate du décès peut mener à une sous-estimation considérable des décès attribuables à d'autres maladies dont une personne est atteinte

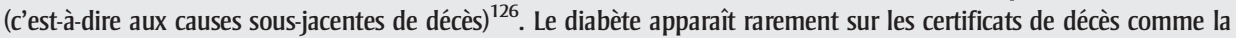
cause immédiate d'un décès parce que les personnes qui en sont atteintes meurent des complications associées au diabète et non de la maladie elle-même. Par exemple, en 2007, le diabète apparaissait comme la principale cause de décès sur seulement 3,1 \% des certificats de décès émis au Canada cette année-là. Pourtant, pour l'année 2008-2009, plus du quart $(29,9 \%)$ de toutes les personnes décédées étaient atteintes de diabète ${ }^{127}$. Le taux de mortalité toutes causes confondues peut plutôt être utilisé pour estimer ce taux de mortalité chez les personnes atteintes de diabète et chez celles qui ne le sont pas, et ce, indépendamment de la cause immédiate du décès inscrite par le médecin sur le certificat de décès.

Mesure

Ratio des taux de mortalité toutes causes confondues au sein de l'ensemble de la population âgée de 20 ans et plus : personnes atteintes du diabète / personnes non atteintes du diabète.

Définition

Le taux de mortalité toutes causes confondues chez les personnes atteintes du diabète est comparé à celui des personnes non atteintes de diabète, ce qui permet de mesurer la surmortalité associée au diabète.

Source des données

Population

Système canadien de surveillance des maladies chroniques (SCSMC), Agénce de la santé publique du Canada.

Population âgée de 20 ans et plus.

Les calculs ont été réalisés en divisant le taux de mortalité toutes causes de décès confondues chez les personnes répondant aux critères établis d'un diabète diagnostiqué par le taux de mortalité toutes causes de décès confondues chez les personnes ne répondant pas aux critères établis d'un diabète diagnostiqué.

Numérateur : Taux de mortalité des personnes atteintes de diabète pour une année (taux de mortalité avec diabète). Dénominateur : Taux de mortalité des personnes non atteintes de diabète pour une année (taux de mortalité sans diabète).

Remarques supplémentaires
Tous les taux présentés sont des taux bruts, à l'exception des tendances pour lesquelles les taux sont normalisés en fonction de l'âge pour tenir compte du Recensement du Canada de 1991. On peut généralement interpréter un rapport de taux de presque 1 comme un résultat positif. 


\section{Mortalité prématurée (années potentielles de vie perdues)}

Justification

Mesure

Définition

Source des données

Population

Méthodes de calcul

Remarques supplémentaires

La mesure des années potentielles de vie perdues (APVP) est largement utilisée pour évaluer la mortalité prématurée (décès précoce) et constitue un important indicateur de l'état de santé général d'une population ${ }^{128}$. L'APVP représente le nombre total d'années que n'aura pas vécues une personne décédée prématurément, soit avant d'atteindre un âge établi ${ }^{124}$. Les décès chez les jeunes ont une plus grande incidence sur l'APVP que ceux chez les aînés. D’après de nombreuses études, le taux de mortalité prématurée constitue un indicateur sensible de l'efficacité des mesures visant à prévenir les maladies chroniques comme le cancer ou les maladies cardiovasculaires ${ }^{129,130}$.

APVP en raison de la cause (ou des causes) ayant entraîné le décès.

On mesure le nombre d'APVP lorsqu'une personne meurt " prématurément » (c.-à-d. avant l'âge de 75 ans) en raison d'une cause ou de plusieurs causes* par 100000 habitants, pour une année donnée. .

Statistique de l'état civil (SEC), Statistique Canada.

Population totale.

Les APVP sont calculées selon chaque groupe d'âge (moins de 1 an, 1 à 4 ans, 5 à 9 ans, ..., 70 à 74 ans) en multipliant le nombre de décès par la différence entre l'âge de 75 ans et la moyenne d'âge au décès dans chacun des groupes d'âge. Elles correspondent à la somme des produits obtenus pour chaque groupe d'âge. Elles sont obtenues en divisant le total des années potentielles de vie perdues par la population totale ayant moins de 75 ans $^{131}$.

${ }^{*}$ Causes particulières de mortalité : maladies cardiovasculaires, cancer, maladies respiratoires chroniques et suicide Les APVP représentent une mesure de la mortalité prématurée dans une population.

Tous les taux présentés sont des taux bruts, à l'exception des tendances pour lesquelles les taux sont normalisés en fonction de l'âge pour tenir compte du Recensement du Canada de 1991.

On peut interpréter une proportion faible pour cet indicateur comme un résultat positif.

\section{Mortalité prématurée (probabilité de mourir)}

Justification

Mesure

Définition

Source des données

Population

Méthodes de calcul

Remarques supplémentaires
En mai 2012, l'Organisation mondiale de la santé a fixé à 25 \% l'objectif concernant la réduction, d'ici 2025, de la mortalité associée aux maladies non transmissibles. Cela doit correspondre aux résultats des efforts de prévention ainsi que de l'augmentation de l'accès aux ressources qui permettent de contrôler et de guérir les maladies non transmissibles. Selon l'Organisation mondiale de la santé, sur les 57 millions de décès survenus dans le monde en 2008, environ 36 millions (63\%) étaient dus à des maladies non transmissibles, dont 14,2 millions de décès prématurés entre l'âge de 30 et de 69 ans ${ }^{132}$. Les données montrent que les décès les plus prématurés liés à des maladies chroniques pourraient être évités ${ }^{133,134}$.

Probabilité de mourir (\%) entre 30 et 69 ans d'une ou de plusieurs maladies chroniques*.

La mortalité prématurée attribuable aux principales maladies chroniques mesure la probabilité inconditionnelle de mourir de façon précoce (entre 30 ans et 69 ans) de l'une des quatre maladies chroniques* principales. Elle est exprimée sous forme d'un pourcentage.

Statistique de l'état civil (SEC), Statistique Canada.

Population totale.

Cet indicateur est calculé à partir de taux de mortalité selon l'âge pour chaque groupe de maladies* de même que pour les catégories de maladies chroniques combinées. La méthode de la table de survie est utilisée pour calculer la probabilité inconditionnelle de mourir $\left(q_{70}^{30}\right)$ entre l'âge de 30 et de 70 ans toutes causes confondues, en l'absence d'autres causes de décès. La méthode de la table de survie de Chiang a servi à calculer q 30/70.

*Maladies chroniques : maladies cardiovasculaires, cancer, diabète et maladies respiratoires chroniques. Le calcul a été réalisé pour chacun des groupes de maladies séparément ainsi que pour leurs combinaisons.

Les estimations en ce qui concerne le diabète sont sans doute en deçà de la réalité, puisque la maladie est rarement considérée comme la cause immédiate de décès ${ }^{127}$.

Cet indicateur correspond à l'indicateur de mortalité prématurée de l'OMC ${ }^{135}$.

On peut interpréter une proportion faible de cet indicateur comme un résultat positif. 


\section{Références}

1. Mikkonen J, Raphael D. Social determinants of health: the Canadian facts. Toronto (Ont.) : York University School of Health Policy and Management; 2010.

2. Wilkinson R, Marmot M (dir.). Les déterminants sociaux de la santé : Les faits. $2^{\mathrm{e}}$ éd. Genève $(\mathrm{CH})$ : Organisation mondiale de la santé; 2003.

3. Statistique Canada. Les seuils de faible revenu [Internet]. Ottawa (Ont.) : Statistique Canada; [consultation le 24 juin 2013]. Consultable en ligne à la page : http://www.statcan.gc.ca /pub/75f0002m/2012002/lico-sfr-fra.htm

4. Whincup PH, Kaye SJ, Owen CG et collab. Birthweight and risk of type 2 diabetes: a systematic review. JAMA. 2008;300(24):2886-97. DOI: $10.1001 /$ jama.2008.886

5. Singhal A, Wells J, Cole TJ, Fewtrell M, Lucas A. Programming of lean body mass: a link between birth weight, obesity and cardiovascular disease. Am J Clin Nutr. 2003;77(3):726-30.

6. Parsons TJ, Power C, Manor O. Fetal and early life growth and body mass index from birth to early adulthood in 1958 British cohort: longitudinal study. BMJ. 2001;323(7325):1331-5.

7. UNICEF, Organisation mondiale de la santé. Low birthweight: country, regional and global estimates. New York (NY) : UNICEF; 2004.

8. Agence de la santé publique du Canada. Rapport sur la santé périnatale au Canada édition 2008. Ottawa (Ont.) : Agence de la santé publique du Canada; 2008.

9. Santé Canada, Société canadienne de pédiatrie, Diététistes du Canada et collab. La nutrition du nourrisson né à terme et en santé : Recommandations de la naissance à six mois [Internet]. Ottawa (Ont.) : Santé Canada; 2007 [consultation le 23 juin 2013]. Consultable en ligne à la page : http://www.hc-sc.gc.ca/fn-an /nutrition/infant-nourisson/recom/index-fra.php

10. Organisation mondiale de la santé, UNICEF. Stratégie mondiale pour l'alimentation du nourrisson et du jeune enfant. Genève $(\mathrm{CH})$ : Organisation mondiale de la santé; 2003.
11. Labayen I, Ruiz JR, Ortega FB et collab. Exclusive breastfeeding duration and cardiorespiratory fitness in children and adolescents. Am J Clin Nutr. 2012;95(2):498-505. DOI: 10.3945 /ajen.111.023838

12. Evelein AM, Geerts CC, Visseren FL et collab. The association between breastfeeding and the cardiovascular system in early childhood. Am J Clin Nutr. 2011;93(4):712-8. DOI: 10.3945/ajcn .110 .002980

13. Horta BL, Bahl R, Martinés JC, Victora CG Evidence on the long-term effects of breastfeeding: systematic reviews and meta-analyses. Genève $(\mathrm{CH})$ : Organisation mondiale de la santé; 2007.

14. Monasta L, Batty GD, Cattaneo A et collab. Early-life determinants of overweight and obesity: a review of systematic reviews. Obes Rev. 2010;11(10):695-708. DOI: 10.1111/j.1467-789X .2010.00735.x

15. Robinson RJ. Carcinogen specific dosimetry model for passive smokers of various ages. Sci Total Environ. 2005;338(3):201-12.

16. Treyster Z, Gitterman B. Second hand smoke exposure in children: environmental factors, physiological effects, and interventions within pediatrics. Rev Environ Health. 2011; 26(3):187-95

17. Tager IB. The effects of second-hand and direct exposure to tobacco smoke on asthma and lung function in adolescence. Paediatr Respir Rev. 2008;9(1):29-37; quiz 37-8. DOI: 10.1016/j.prrv.2007.11.006

18. Santé Canada. Le tabagisme et votre corps [Internet]. Ottawa (Ont.) : Santé Canada; [modifié le $1^{\mathrm{er}}$ nov. 2011; consultation le 24 juin 2013]. Consultable en ligne à la page : http://www.hc-sc .gc.ca/hc-ps/tobac-tabac/body-corps/index-fra .php

19. Warburton DE, Charlesworth S, Ivey A, Nettlefold L, Bredin SS. A systematic review of the evidence for Canada's Physical Activity Guidelines for Adults. Int J Behav Nutr Phys Act. 2010;7:39. DOI: 10.1186/1479-5868-7-39

20. Reddigan JI, Ardern CI, Riddell MC, Kuk JL. Relation of physical activity to cardiovascular disease mortality and the influence of cardiometabolic risk factors. Am J Cardiol. 2011; 108(10):1426-31. DOI: 10.1016/j.amjcard.2011 .07 .005
21. Cumming TB, Tyedin K, Churilov L, Morris ME, Bernhardt J. The effect of physical activity on cognitive function after stroke: a systematic review. Int Psychogeriatr. 2011;24(4):557-67.

22. Winzer BM, Whiteman DC, Reeves MM, Paratz JD. Physical activity and cancer prevention: a systematic review of clinical trials. Cancer Causes Control. 2011;22(6):811-26.

23. Jeon CY, Lokken RP, Hu FB, van Dam RM. Physical activity of moderate intensity and risk of type 2 diabetes: a systematic review. Diabetes Care. 2007;30(3):744-52.

24. Biddle SJ, Asare M. Physical activity and mental health in children and adolescents: a review of reviews. Br J Sports Med. 2011; 45(11):886-95.

25. Penedo FJ, Dahn JR. Exercise and wellbeing: a review of mental and physical health benefits associated with physical activity. Curr Opin Psychiatry. 2005;18(2):189-93.

26. Craig CL, Cameron C, Tudor-Locke C. CANPLAY pedometer normative reference data for 21271 children and 12956 adolescents. Med Sci Sports Exerc. 2013;45(1):123-9. DOI: 10.1249 /MSS.0b013e31826a0f3a

27. Craig CL, Tudor-Locke C, Cragg S, Cameron C. Process and treatment of pedometer data collection for youth: the Canadian Physical Activity Levels among Youth study. Med Sci Sports Exerc. 2010;42(3):430-5. DOI: 10.1249 /MSS.0b013e3181b67544

28. Société canadienne de physiologie de l'exercice (SCPE). Directives canadiennes en matière d'activité physique à l'intention des enfants âgés de 5 à 11 ans et des jeunes âgés de 12 à 17 ans. Ottawa (Ont.) : SCPE; [consultation le 23 juin 2013]. Consultable en ligne à la page : http:// www.csep.ca/francais/view.asp? $\mathrm{x}=804 \#$

29. Colley RC, Janssen I, Tremblay MS. Daily step target to measure adherence to physical activity guidelines in children. Med Sci Sports Exerc. 2012;44(5):977-82. DOI: 10.1249/MSS $.0 \mathrm{~b} 013 \mathrm{e} 31823 \mathrm{f} 23 \mathrm{~b} 1$

30. Hamburg NM, McMackin CJ, Huang AL et collab. Physical inactivity rapidly induces insulin resistance and microvascular dysfunction in healthy volunteers. Arterioscler Thromb Vasc Biol. 2007;27(12):2650-6. 
31. Hamilton MT, Hamilton DG, Zderic TW. Role of low energy expenditure and sitting in obesity, metabolic syndrome, type 2 diabetes, and cardiovascular disease. Diabetes. 2007; 56(11):2655-67.

32. Katzmarzyk PT, Church TS, Craig CL, Bouchard, C. Sitting time and mortality from all causes, cardiovascular disease, and cancer. Med Sci Sports Exerc. 2009;41(5):998-1005.

33. Shields M, Tremblay MS. Comportements sédentaires et obésité. Rapports sur la santé. 2008;19(2):19-33.

34. Stamatakis E, Hamer M, Dunstan DW. Screen-based entertainment time, all-cause mortality, and cardiovascular events: populationbased study with ongoing mortality and hospital events follow-up. J Am Coll Cardiol. 2011; 57(3):292-9.

35. Edwardson CL, Gorely T, Davies MJ et collab. Association of sedentary behaviour with metabolic syndrome: a meta-analysis. PLoS One. 2012;7:e34916.

36. Tremblay MS, LeBlanc AG, Janssen I et collab. Canadian sedentary behaviour guidelines for children and youth. Appl Physiol Nutr Metab. 2011;36(1):59-64;65-71. DOI: 10.1139 /H11-012

37. Stamatakis E, Hamer M, Dunstan DW. Screen-based entertainment time, all-cause mortality, and cardiovascular events JACC. 2011;57(3):292-9.

38. Martin LJ, Lee SY, Couch SC, Morrison J, Woo JG. Shared genetic contributions of fruit and vegetable consumption with BMI in families $20 \mathrm{y}$ after sharing a household. Am J Clin Nutr. 2011;94:1138-43.

39. Alinia S, Hels O, Tetens I. The potential association between fruit intake and body weight--a review. Obes Rev. 2009;10(6):639-47. DOI: 10.1111/j.1467-789X.2009.00582.X

40. Mirmiran P, Noori N, Zavareh MB, Azizi F. Fruit and vegetable consumption and risk factors for cardiovascular disease. Metabolism. 2009;58(4):460-8. DOI: 10.1016/j.metabol.2008 .11 .002

41. Ford ES, Mokdad AH. Fruit and vegetable consumption and diabetes mellitus incidence among U.S. adults. Prev Med. 2001;32(1):33-9.
42. Wirt A, CE Collins. Diet quality-what is it and does it matter? Public Health Nutr. 2009;12(12):2473-92.

43. Collins CE, Young AF, Hodge A. Diet quality is associated with higher nutrient intake and selfrated health in mid-aged women. J Am Coll Nutr. 2008;27(1):146-57.

44. McNaughton SA, Bates CJ, Mishra GD. Diet quality is associated with all-cause mortality in adults aged 65 years and older. J Nutr. 2012; 142(2):320-5. DOI: 10.3945/jn.111.148692

45. Garriguet D. La qualité de l'alimentation au Canada. Rapports sur la santé. 2009;20(3):1-13.

46. Traynor MM, Holowaty PH, Reid DJ, GrayDonald K. Vegetable and fruit food frequency questionnaire serves as a proxy for quantified intake. Can J Public Health. 2006;97(4):286-90.

47. Santé Canada. Bien manger avec le Guide alimentaire canadien [Internet]. Ottawa (Ont.) : Santé Canada; [modifié le 23 mai 2013; consultation le 9 janvier 2014]. Téléchargeable à partir du lien : http://www.hc-sc.gc.ca/fn-an /alt_formats/hpfb-dgpsa/pdf/food-guide-aliment /view_eatwell_vue_bienmang-fra.pdf

48. Malik V, Popkin B, Bray G, Després JP, Hu FB. Sugar-sweetened beverages, obesity, type 2 diabetes mellitus, and cardiovascular disease risk. Circulation. 2010;121(11):1356-64. DOI: 10.1161/CIRCULATIONAHA.109.876185

49. Organisation mondiale de la santé. Consommation d'alcool - février 2011 [Internet]. Genève $(\mathrm{CH})$ : Organisation mondiale de la santé; [consultation le 24 janvier 2013]. Consultable en ligne à la page : http:// www.who.int/mediacentre/factsheets/fs349/fr /index.html

50. Rehm J, Baliunas D, Borges GL et collab. The relation between different dimensions of alcohol consumption and burden of disease: an overview. Addiction. 2010;105(5):817-43. DOI: 10.1111/j.1360-0443.2010.02899.x

51. Butt P, Beirness D, Gliksman L, Paradis C, Stockwell T. L'alcool et la santé au Canada : résumé des données probantes et des directives de consommation à faible risque. Ottawa (Ont.) : Centre canadien de lutte contre les toxicomanies; 2011.
52. Cohen S, Janicki-Deverts D, Doyle WJ et collab. Chronic stress, glucocorticoid receptor resistance, inflammation, and disease risk. Proc Natl Acad Sci USA. 2012;109(16):5995-9. DOI: 10.1073/pnas.1118355109

53. Szczepanska-Sadowska E, CudnochJedrzejewska A, Ufnal M, Zera T. Brain and cardiovascular diseases: common neurogenic background of cardiovascular, metabolic and inflammatory diseases. J Physiol Pharmacol. 2010;61(5):509-21.

54. Bauer ME, Jeckel CM, Luz C. The role of stress factors during aging of the immune system. Ann N Y Acad Sci. 2009;1153:139-52. DOI: 10.1111 /j.1749-6632.2008.03966.x

55. Steptoe A, Kivimaki M. Stress and cardiovascular disease. Nat Rev Cardiol. 2012;9(6):360-70. DOI: $10.1038 /$ nrcardio.2012.45

56. Menezes AR, Lavie CJ, Milani RV, O’Keefe J, Lavie TJ. Psychological risk factors and cardiovascular disease: is it all in your head? Postgrad Med. 2011;123(5):165-76. DOI: 10.3810/pgm .2011 .09 .2472

57. Barlow SE, Expert Committee. Expert committee recommendations regarding the prevention, assessment, and treatment of child and adolescent overweight and obesity: summary report. Pediatrics. 2007;120 Suppl 4:S164-92.

58. Nguyen T, Lau DC. The obesity epidemic and its impact on hypertension. Can J Cardiol. 2012;28(3):326-33. DOI: 10.1016/j.cjca.2012.01 .001

59. Garber AJ. Obesity and type 2 diabetes: which patients are at risk? Diabetes Obes Metab. 2012;14(5):399-408. DOI: $10.1111 / \mathrm{j}$ .1463-1326.2011.01536.x

60. Aguilar Cordero MJ, Gonzalez JE, Garcia Lopez AP et collab. [Obesity and its implication in breast cancer]. Nutr Hosp. 2011;26(4):899903. DOI: $10.1590 / S 0212-16112011000400033$

61. Janssen I, Katzmarzyk PT, Srinivasan SR et collab. Utility of childhood BMI in the prediction of adulthood disease: Comparison of national and international references. Obes Res. 2005;13(6):1106-15.

62. Steinberger J, Daniels SR, Obesity, insulin resistance, diabetes, and cardiovascular risk in children. Circulation. 2003;107(10):1448-53. 
63. De Onis M, Onyango AW, Borghi E, Siyam A, Nishida C, Siekmann J. Development of a WHO growth reference for school-aged children and adolescents. Bull World Health Organ. 2007; 85(9):660-7.

64. Fowler MJ. Microvascular and macrovascular complications of diabetes. Clin Diabetes. 2008;26(2):77-82. DOI: 10.2337/diaclin.26.2.77

65. Milman S, Crandall JP. Mechanisms of vascular complications in prediabetes. Med Clin North Am. 2011 Mar;95(2):309-25, vii. DOI: $10.1016 /$ j.mcna.2010.11.004

66. Nwose EU, Richards RS, McDonald S et collab. Assessment of diabetic macrovascular complications: a prediabetes model. $\mathrm{Br} \mathrm{J}$ Biomed Sci. 2010;67(2):59-66.

67. Plutzky J. Macrovascular effects and safety issues of therapies for type 2 diabetes. Am J Cardiol. 2011;108(3 Suppl):25B-32B.

68. American Diabetes Association. Standards of medical care in diabetes-2011. Diabetes Care. 2011;34 Suppl 1:S11-61. DOI: 10.2337/dc11-S011

69. Ali MK, Bullard KM, Imperatore G et collab. Characteristics associated with poor glycemic control among adults with self-reported diagnosed diabetes - national health and nutrition examination survey, United States, 2007-2010. MMWR Morb Mortal Wkly Rep. 2012;61 Suppl:32-7.

70. Jacobson AM. Impact of improved glycemic control on quality of life in patients with diabetes. Endocr Pract. 2004;10(6):502-8.

71. American Diabetes Association. Standards of medical care in diabetes-2011. Diabetes Care. 2011;34 Suppl 1:S11-61. DOI: 10.2337/dc11-S011

72. Lloyd-Jones DM, Leip EP, Larson MG et collab. Novel approach to examining first cardiovascular events after hypertension onset. Hypertension. 2005;45(1):39-45.

73. Lewington S, Clarke R, Qizilbash N, Peto R, Collins R. Age-specific relevance of usual blood pressure to vascular mortality: a meta-analysis of individual data for one million adults in 61 prospective studies. Lancet. 2002;360(9349): 1903-13.

74. Morrison AC, Ness RB. Sodium intake and cardiovascular disease. Annu Rev Public Health. 2011;32:71-90. DOI: 10.1146/annurev-publhealth $-031210-101209$
75. Ignarro LJ, Balestrieri ML, Napoli C. Nutrition, physical activity, and cardiovascular disease: an update. Cardiovasc Res. 2007;73(2): 326-40.

76. Hypertension Canada. Programme éducatif canadien sur l'hypertension (PECH) Recommandations 2013 [Internet]. Markham (Ont.) : Hypertension Canada; [consultation le 23 juin 2013]. Consultable en ligne à la page : http:// www.hypertension.ca/fr/chep

77. Chobanian AV, Bakris GL, Black HR et collab. The seventh report of the Joint National Committee on Prevention, Detection, Evaluation, and Treatment of High Blood Pressure: the JNC 7 report. JAMA. 2003;289(19):2560-72.

78. Tian L, Fu M. The relationship between high density lipoprotein subclass profile and plasma lipids concentrations. Lipids Health Dis. 2010;9:118. DOI: 10.1186/1476-511X-9-118

79. Aryal M, Poudel A, Satyal B et collab. Evaluation of non-HDL-c and total cholesterol: HDL-c Ratio as cumulative marker of cardiovascular risk in diabetes mellitus. Kathmandu Univ Med J (KUMJ). 2010;8(32):398-404.

80. Nair D, Carrigan TP, Curtin RJ et collab. Association of total cholesterol/ high-density lipoprotein cholesterol ratio with proximal coronary atherosclerosis detected by multislice computed tomography. Prev Cardiol. 2009; 12(1):19-26.

81. Kannel WB, Vasan RS, Keyes MJ, Sullivan LM, Robins SJ. Usefulness of the triglyceridehigh-density lipoprotein versus the cholesterolhigh-density lipoprotein ratio for predicting insulin resistance and cardiometabolic risk (from the Framingham Offspring Cohort). Am J Cardiol. 2008;101(4):497-501. DOI: 10.1016/j .amjcard.2007.09.109

82. Manuel DG, Tanuseputro P, Mustard CA et collab. The 2003 Canadian recommendations for dyslipidemia management. CMAJ. 2005; 172(8):1027-31.

83. Genest J, McPherson R, Frohlich J et collab. Canadian Cardiovascular Society/Canadian guidelines for the diagnosis and treatment of dyslipidemia and prevention of cardiovascular disease in the adult - 2009 recommendations. Can J Cardiol. 2009;25(10):567-79.

84. Manuel DG, Tanuseputro P, Mustard CA et collab. The 2003 Canadian recommendations for dyslipidemia management: revisions are needed. CMAJ. 2005;172(8):1027-31.
85. Si D, Bailie R, Cunningham $\mathrm{J}$ et collab. Describing and analysing primary health care system support for chronic illness care in Indigenous communities in Australia's Northern Territory - use of the Chronic Care Model. BMC Health Serv Res. 2008;8:112. DOI: 10.1186/1472-6963-8-112

86. Towards a national primary health care strategy: a discussion paper from the Australian government. Canberra (AU): Australian Government, Department of Health and Ageing; 2008.

87. L'Association dentaire canadienne. Exposé de position de l'Association dentaire canadienne (ADC : L'accès aux soins buccodentaires pour les Canadiens. Ottawa (Ont.) : L'Association dentaire canadienne; 2010.

88. Agence de la santé publique du Canada. Programmes organisés de dépistage du cancer du sein au Canada : rapport sur le rendement des programmes en 2005 et en 2006. Ottawa (Ont.) : Agence de la santé publique du Canada; 2011. (n॰ HP32-1/2006E-PDF)

89. Doyle GP, Major D, Chu C et collab. Étude sur la participation à la mammographie de dépistage et sur son utilisation au Canada. Maladies chroniques et blessures au Canada. 2011;31(4):154-159.

90. Greif JM. Mammographic screening for breast cancer: an invited review of the benefits and costs. Breast. 2010;19(4):268-72. DOI: 10.1016/j.breast.2010.03.017

91. Canadian Task Force on Preventive Health Care, Tonelli M, Connor Gorber S et collab. Recommendations on screening for breast cancer in average-risk women aged 40-74 years. CMAJ. 2011;183(17):1991-2001. DOI: 10.1503/cmaj .110334

92. Groupe d'étude canadien sur les soins de santé préventifs. Dépistage du cancer du sein : résumé des recommandations à l'intention des cliniciens et des décideurs [Internet]. Ottawa (Ont.) : Groupe d'étude canadien sur les soins de santé préventifs; [consultation le 24 juin 2013]. Consultable en ligne à la page : http://canadian taskforce.ca/fr/guidelines/2011-breast-cancer/

93. Groupe de travail sur les indicateurs de rendement en matière de dépistage, Réseau de prévention et de contrôle du cancer du col utérin (RPCCCU). Surveillance du rendement des programmes de dépistage du cancer du col utérin au Canada. Ottawa (Ont.) : Agence de la santé publique du Canada; 2009. 
94. Spence AR, Goggin P, Franco EL. Process of care failures in invasive cervical cancer: systematic review and metaanalysis. Prev Med. 2007;45(2-3):93-106.

95. Groupe d'étude canadien sur les soins de santé préventifs. Dépistage du cancer du col de l'utérus : résumé des recommandations pour les cliniciens et les responsables des politiques [Internet]. Ottawa (Ont.) : Groupe d'étude canadien sur les soins de santé préventifs; [consultation le 24 juin 2013]. Consultable en ligne à la page : http://canadiantaskforce.ca /fr/guidelines/screening-for-cervical-cancer/

96. Société canadienne du cancer. Comité directeur des statistiques sur le cancer. Toronto (Ont.) : Société canadienne du cancer; 2012.

97. Bretthauer M. Colorectal cancer screening. J Intern Med. 2011;270(2):87-98. DOI: 10.1111/j $.1365-2796.2011 .02399 . x$

98. Joanne M. Langley, Marie E. Faughnan, and the Canadian Task Force on Preventive Health Care. Prevention of influenza in the general population: recommendation statement from the Canadian Task Force on Preventive Health Care. CMAJ. 2004;171(10):1213-22.

99. Heymann AD, Shapiro Y, Chodick G et collab. Reduced hospitalizations and death associated with influenza vaccination among patients with and without diabetes. Diabetes Care. 2004;27(11):2581-4.

100. Comité consultatif national de l'immunisation. Déclaration sur la vaccination antigrippale pour la saison 2011-2012. Relevé des maladies transmissibles au Canada. 2011;37(ACS-5).

101. Kaplan GA, Camacho T. Perceived health and mortality: a nine-year follow-up of the human population laboratory cohort. Am J Epidemiol. 1983;117(3):292-304.

102. Dogra S. Better self-perceived health is associated with lower odds of physical inactivity in older adults with chronic disease. J Aging Phys Act. 2011;19(4):322-35.

103. Barreto SM, Figueiredo RC. Chronic diseases, self-perceived health status and health risk behaviors: gender differences. Rev Saude Publica. 2009;43 Suppl 2:38-47.
104. Organisation mondiale de la santé. Qu'est-ce que la santé mentale? [Internet]. Ottawa (Ont.) : Organisation mondiale de la santé (2007); [consultation en janvier 2014]. Consultable en ligne à la page : http://www.who.int/features/qa/62/fr /index.html

105. Cott CA, Gignac MA, Badley EM. Determinants of self rated health for Canadians with chronic disease and disability. J Epidemiol Community Health. 1999;53(11): 731-6.

106. Shields M, Shooshtari S. Déterminants de l'autoévalutation de la santé. Rapports sur la santé. 2001;13(1):39-63.

107. Mawani H, Gilmour H. Validation de l'autoévaluation de la santé mentale. Rapports sur la santé. 2010;21(3):1-16. Statistique Canada (n॰82-003-XPF)

108. Fang R, Millar JS. Canada's global position in life expectancy: a longitudinal comparison with the healthiest countries in the world. Can J Public Health. 2009;100(1):9-13.

109. Statistique Canada. Espérance de vie [Internet]. Ottawa (Ont.) : Statistique Canada; [consultation le 24 juin 2013]. Consultable en ligne à la page : http://www.statcan.gc.ca/pub /82-229-x/2009001/demo/lif-fra.htm

110. Chiang CL. The life table and its applications. Malabar (FL): Robert E. Krieger Publishing Company; 1984.

111. Hsieh JJ. A general theory of life table construction and a precise abridged life table method. Biom J. 1991;33(2):143-62.

112. Loukine L, Waters C, Choi BC, Ellison J. Health-adjusted life expectancy among Canadian adults with and without hypertension. Cardiol Res Pract. 2011;2011:612968. DOI: 10.4061 /2011/612968.

113. Statistique Canada. 37-HLT Espérance de vie ajustée en fonction de la santé (EVAS) [Internet]. Ottawa (Ont.) : Statistique Canada; [consultation le 24 juin 2013]. Consultable en ligne à la page : http://www.statcan.gc .ca/pub/82-401-x/2006000/considerations/hlt /4064328-fra.htm

114. Sullivan DF. A single index of mortality and morbidity. HSMHA Health Rep. 1971; 86(4):347-54.

115. Gordis L. Epidemiology. $3^{\mathrm{e}}$ éd. Philadelphia: Elsevier Saunders; 2004.
116. Aschengrau A, Seage GR. Essentials of epidemiology in public health. Sudbury (MA): Jones and Bartlett Publishers; 2003.

117. Boyd C, Fortin M. Future of multimorbidity research: how should understanding of multimorbidity inform health system design. Public Health Rev. 32(2):451-74.

118. Conseil canadien de la santé. Schémas de population - maladies chroniques au Canada. Supplément de données à Importance du renouvellement des soins de santé : à l'écoute des Canadiens atteints de maladies chroniques. Toronto (Ont.) : Conseil canadien de la santé; 2007.

119. Klijs B, Nusselder WJ, Looman CW, Mackenbach JP. Contribution of chronic disease to the burden of disability. PLoS One. 2011;6(9):e25325. DOI: 10.1371/journal.pone .0025325

120. Puts MT, Deeg DJ, Hoeymans N et collab. Changes in the prevalence of chronic disease and the association with disability in the older Dutch population between 1987 and 2001. Age Ageing. 2008;37(2):187-93. DOI: 10.1093/ageing /afm185

121. Gadalla T. Association des troubles de l'humeur concomitants et des maladies chroniques avec l'incapacité et la qualité de vie en Ontario, Canada. Maladies chroniques au Canada. 2008;28(4):166-173.

122. Bhattacharya J, Choudhry K, Lakdawalla D. Chronic disease and severe disability among working-age populations. Med Care. 2008; 46(1):92-100.

123. Mathers $C D$, Fat $D M$, Inoue $M$, Rao $C$, Lopez AD. Counting the dead and what they died from: an assessment of the global status of cause of death data. Bull World Health Organ. 2005;83(3):171-7. Epub 2005 Mar 16.

124. Organisation mondiale de la santé. WHO Stat Definitions and Metadata 2006 [Internet]. Genève $(\mathrm{CH})$ : Organisation mondiale de la santé; [consultation le 24 juin 2013]. Téléchargeable à partir du lien : http://www .who.int/whosis/whostat2006DefinitionsAnd Metadata.pdf

125. Australian Institute of Health and Welfare. Health and wellbeing of young Australians: indicator framework and key national indicators. Canberra (AU): AIHW; AIHW bulletin $n^{\circ}$ 77; 2010. (n॰ AUS 123) 
126. Coeli CM, Ferreira LG, Drbal Md Mde M, et collab. Diabetes mellitus mortality among elderly as an underlying or secondary cause of death. Rev Saude Publica. 2002;36(2):135-40.

127. Agence de la santé publique du Canada. Le diabète au Canada : Perspective de santé publique sur les faits et chiffres. Ottawa (Ont.) : Agence de la santé publique du Canada; 2011.

128. Healthy People 2020: General health status [Internet]. Washington (DC): US Department of Health and Human Services; [consultation le 24 juin 2013]. Consultable en ligne à la page : http:// healthypeople.gov/2020/about/GenHealthAbout .aspx

129. Fukuda Y, Nakamura K, Takano T. Turning points in time trends of cancer mortality in Japan: premature mortality is more sensitive in the progress of cancer prevention. Environ Health Prev Med. 2001;5(4):155-9. DOI: 10.1007 /BF02918292

130. Hennekens $\mathrm{CH}$. Prevention of premature mortality among patients with schizophrenia: the need for primary prevention efforts in cardiovascular disease. CNS Spectr. 2008;13(6 Suppl 10):9-10.

131. Association of Public Health Epidemiologists in Ontario. APHEO core indicators, PYLL, age group method [Internet]. Ontario : APHEO; [consultation le 24 juin 2013]. Consultable en ligne à la page : http:// www.apheo.ca/index.php?pid $=190$

132. Organisation mondiale de la santé. Cadre mondial de suivi, comprenant des indicateurs, et série de cibles mondiales volontaires pour la lutte contre les maladies non transmissibles. Document OMS de réflexion révisé (version du 25 juillet 2012). Genève (CH) : Organisation mondiale de la santé; 2012.

133. Australian Institute of Health and Welfare. Premature mortality from chronic disease. AIHW Bulletin. 2010 Dec; 84.

134. Robles S, Adrion E, Anderson GF. Premature adult mortality from non-communicable diseases in three middle-income countries: do NCD programmes matter? Health Policy Plan. 2012;27 (6):487-98. DOI: 10.1093/heapol/czr073 .20
135. Organisation mondiale de la santé. Mortality from NCDs [Internet]. Genève $(\mathrm{CH})$ : Organisation mondiale de la santé; [consultation le 24 juin 2013]. Téléchargeable à partir du lien : http://www.who.int/nmh/events/2012/note _20120426.pdf 


\section{$M C B C$ : Information pour les auteurs}

\section{Notre revue \\ Mandat de $\mathrm{MCBC}$}

Maladies chroniques et blessures au Canada (MCBC) est une revue scientifique trimestrielle qui traite de la prévention des maladies non transmissibles et des blessures au Canada ainsi que de la lutte contre celles-ci. Ses articles de fond sont soumis à une évaluation par les pairs. Le contenu des articles peut couvrir des projets de recherche en épidémiologie, en équité en santé, en santé publique ou communautaire, en biostatistique, en sciences du comportement, en services de santé et en économie de la santé. $M C B C$ favorise la communication sur les ma-ladies chroniques et les blessures entre professionnels de la santé publique, épidémiologistes et chercheurs, planificateurs de politiques de la santé et éducateurs en santé. Les articles sont choisis en fonction de leur qua-lité scientifique, de leur pertinence en santé publique, de leur clarté, de leur concision et de l'exactitude technique. Même si $M C B C$ est une publication de l'Agence de la santé publique du Canada (ASPC), les contributions de membres ne travaillant pas pour le gouvernement fédéral sont les bienvenues.

\section{Pourquoi publier des articles dans MCBC?}

Étant donné que la revue est en libre accès et qu'elle est totalement bilingue, elle est consultée par des lecteurs des États-Unis, d'Europe et d'Afrique francophone. $M C B C$ jouit d'une forte présence en ligne parce qu'elle est répertoriée, entre autres, par Index Medicus/MEDLINE (Pubmed), Journal Citation Reports/ Science Edition (Thomson Reuters), Elsevier, CAB Abstracts/Global Health, SciVerse Scopus, la Bibliothèque virtuelle canadienne de la santé, SciSearch, EBSCO, ProQuest et MediaFinder. La revue est une plateforme importante d'échange de connaissances au sein de la communauté de la santé publique au Canada.

\section{Types d'articles}

Article de fond évalué par des pairs:

Article de recherche quantitative: Article dont le corps du texte compte un maximum de 3500 mots en anglais, (4 400 mots en français) (excluant le résumé, les tableaux, les figures et les références), présenté sous forme de recherche originale, de rapports de surveillance, d'examens systématiques (notamment des méta-analyses) ou d'articles méthodologiques. Un résumé structuré de 250 mots maximum en anglais (345 mots en français) doit être inclus.

Article de recherche qualitative : Article dont le corps du texte compte un maximum de 5000 mots en anglais (6 500 mots en français) (excluant le résumé, les tableaux, les figures et les références). Un résumé structuré de 250 mots maximum en anglais (345 mots en français) doit être inclus. $M C B C$ suit les lignes directrices de Social Science and Medicine concernant les articles de recherche qualitative: http://www.elsevier.com /wps/find/journaldescription.cws_home/315 /authorinstructions.

Rapport d'étape : Description de programmes nationaux, d'études ou de systèmes d'information qui portent sur la santé publique canadienne (maximum de 3000 mots en anglais ou de 3900 mots en français). Un rapport d'étape peut faire l'objet d'une évaluation par les pairs, et le rédacteur en chef peut exiger la présentation d'un résumé.

Forum pancanadien: Conçu pour que les auteurs puissent présenter ou échanger de l'information ou discuter sur des données de surveillance régionale ou nationale, des programmes en cours d'élaboration ou des initiatives liées à des politiques en santé publique (maximum de 3000 mots en anglais ou de 3900 mots en français). Les documents peuvent faire l'objet d'une évaluation par les pairs, et le rédacteur en chef peut exiger la présentation d'un résumé

Rapport d'atelier ou de conférence : Compte rendu d'événements importants concernant la santé publique nationale tenus récemment (maximum de 1200 mots en anglais ou de 1560 mots en français). Résumé non requis.
Note de synthèse : Maximum de 1000 mots en anglais (1 300 mots en français). La note de synthèse permet aux auteurs de littérature grise de voir leurs résultats pertinents apparaître dans PubMed en tant que "nouvelles " ("News"). Résumé non requis.

Compte rendu de livre ou de logiciel : Les comptes rendus sont habituellement proposés par les rédacteurs (maximum de 800 mots en anglais ou 1000 mots en français), mais les offres de compte rendu sont les bienvenues. Résumé non requis.

Lettre au rédacteur en chef : Les commentaires sur des articles récemment publiés dans $M C B C$ sont pris en considération pour publication (maximum de 500 mots en anglais ou de 630 mots en français). Les commentaires doivent être reçus au plus tard un mois après la date de publication de l'article en question pour être pris en considération. Résumé non requis.

\section{Soumettre un manuscrit à $M C B C$}

Veuillez soumettre votre manuscrit au rédacteur en chef de $M C B C$, à cdic-mcbc@phac-aspc.gc.ca.

Étant donné que $M C B C$ se conforme en général aux exigences «Uniform Requirements for Manuscripts Submitted to Biomedical Journals " approuvées pa l'International Committee of Medical Journal Editors, les auteurs devraient consulter ce document (sauf pour les illustrations) pour préparer leur manuscrit à $M C B C$ (voir www.icmje.org). Pour obtenir une feuille de style plus détaillée utilisée par $M C B C$, veuillez communiquer avec la gestionnaire de la rédaction, à cdic-mcbc@phac-aspc gc.ca.

Si un ou plusieurs auteurs sont internes, c'est-àdire qu'ils travaillent pour Santé Canada, l'ASPC, les Instituts de recherche en santé du Canada, le Conseil de contrôle des renseignements relatifs aux matières dangereuses, le Conseil d'examen du prix des médicaments brevetés ou l'Agence canadienne de contrôle de la procréation assistée, l'approbation du gestionnaire de programme et du directeur est requise.

\section{Liste de vérification pour la présentation d'un manuscrit}

Lettre d'accompagnement et conditions de paternité de l'oeuvre : Le document doit être signé par tous les auteurs, lesquels affirment avoir lu et approuvé le manuscrit final. Les auteurs doivent confirmer que le matériel n’a pas été publié ailleurs en partie ou en totalité et que l'article n'est pas en cours d'évaluation pour publication ailleurs. Les auteurs doivent confirmer qu'ils remplissent les conditions de paternité de l'œuvre suivantes: participation à la conception et à la conceptualisation de l'étude, et/ou à l'analys ou à l'interprétation des données et/ou à la rédaction de l'article. Les auteurs doivent signaler tout conflit d’intérêts le cas échéant.

Veuillez envoyer une copie de la lettre signée par télécopieur (613-941-2057) ou par courriel (cdic-mcbc@phac-aspc.gc.ca).

Première page titre : Titre concis; nom au complet, affiliations institutionnelles et diplôme le plus élevé de chaque auteur; nom, adresse postale, courriel, numér de téléphone et numéro de télécopieur des auteurs-ressource (pour la correspondance); décompte de mots du résumé et du corps du texte (pris séparément) numéros des tableaux et des figures.

Deuxième page titre: Titre seulement; démarre la numérotation des pages à partir de celle-ci, soit la page 1 .

Résumé : Texte structuré (introduction, méthodologie, résultats, conclusion) s'il y a lieu; inclure de 3 à 8 mots-clés (préférablement à partir des termes Medical Subject Headings [MeSH] d'Index Medicus).

Texte : En format Microsoft Word, à double interligne, marges d'un pouce $(25 \mathrm{~mm})$, police de caractères de taille 12. Dans le cas d'articles de recherche faisan l'objet d'une évaluation par les pairs, il faut adopter la structure suivante (sections) : Introduction, Méthodologie, Résultats, Analyse/Discussion et Conclusion. La section "Analyse/Discussion" devrait comporte une sous-section "Forces et limites". Pour la conclusion, il faut éviter les énoncés ne correspondant pas aux résultats de l'étude.
Remerciements : Inclure l'aide matérielle et financière; si une personne est remerciée, les auteurs doivent indiquer dans la lettre d'accompagnement qu'ils ont obtenu la permission de cette dernière par écrit.

Références : Respecter le style Vancouver (des exemples se trouvent à : http://www.ncbi.nlm.nih.gov /books/NBK7256/ pour l'anglais; pour le français, utiliser notre feuille de style détaillée ou un numéro récemment paru); nommer jusqu'à six auteurs par référence (trois premiers auteurs, puis " et collab. " s'il y en a plus de six). La numérotation doit figurer en exposant, dans l'ordre suivi dans le texte, les tableaux et les figures. Prière de ne pas utiliser la fonction de numérotation automatique des références du logiciel de traitement de texte ni les inclusions automatiques de logiciels bibliographiques. Pour les observations ou les données non publiées ou encore les communications personnelles utilisées (déconseillées), il faut les indiquer dans le texte, entre parenthèses (les auteurs doivent obtenir la permission par écrit). Les auteurs doivent vérifier l'exactitude des références et des hyperliens

Tableaux et figures : Les tableaux et figures préparés dans Word doivent figurer à la fin du document principal. S'ils ont été préparés dans Excel, il faut les sauvegarder dans un fichier séparé. Ils doivent être aussi explicites et concis que possible, numérotés dans l'ordre dans lequel ils apparaissent dans le texte et les notes explicatives des tableaux doivent être insérés en bas de page et indiquées à l'aide de lettres minuscules (en ordre alphabétique) en exposant. Les figures ne peuvent être que des graphiques, des organigrammes ou diagrammes ou des cartes (aucune photo). Si les figures sont fournies dans le document Word, les données brutes correspondantes seront demandées une fois le manuscrit accepté pour publication.

\section{Processus de révision}

Pour les articles évalués par les pairs : Les articles sont tout d'abord évalués par le rédacteur en chef ains que par un rédacteur scientifique adjoint afin d'en déterminer la pertinence. Si l'article correspond au mandat de $M C B C$, l'article est soumis à une évaluation par les pairs à double insu. Par la suite, le rédacteur scientifique adjoint reçoit les évaluations des pairs, se prononce sur celles-ci puis formule l'une des recommandations suivantes : " accepter »; " réévaluer après modifications mineures "; "réévaluer après modifications majeures » ou « rejeter ». Dans le cas d'un article à réévaluer, les auteurs se verront accorder un temps suffisant pour y apporter les modifications nécessaires et répondre aux commentaires et questions des évaluateurs. Si le manuscrit est accepté après l'évaluation par les pairs, il est nécessaire de suivre notre processus interne de révision avant l'acceptation finale.

Pour les articles non évalués par les pairs : Les articles sont tout d'abord évalués par le rédacteur en chef et, si nécessaire, par un rédacteur scientifique adjoint afin d'en déterminer la pertinence. Si des révisions sont demandées, les auteurs se verront accorder un temps suffisant pour apporter les modifications nécessaires. Si le manuscrit est accepté, il est nécessaire de suivre notre processus interne de révision avant l'acceptation finale.

\section{Droits d'auteur}

L'ASPC demande aux auteurs de céder officiellement par écrit leurs droits d'auteur pour chaque article publié dans la revue $M C B C$. Une fois l'article accepté pour publication, un formulaire de renonciation aux droits d'auteur sera envoyé pour signature.Pour de plus amples renseignements, communiquez avec la gestionnaire de la rédaction, à cdic-mcbc@phac-aspc.gc.ca.

\section{Éthique en matière de publication}

Étant donné que $M C B C$ se conforme en général aux exigences «Uniform Requirements for Manuscripts Submitted to Biomedical Journals " approuvées par l'International Committee of Medical Journal Editors, les auteurs doivent consulter ce document à propos de l'éthique en matière de publication (www.icmje.org). 
